\title{
Electric Load Modeling for Long-Term Forecasting
}

\subsection{Introduction}

Long-term electric peak-load forecasting is an important issue in effective and efficient planning. Over- or underestimation can greatly affect the revenue of the electric utility industry. Overestimation of the future load may lead to spending more money in building new power stations to supply this load. Moreover, underestimation of load may cause troubles in supplying this load from the available electric supplies and produce a shortage in the spinning reserve of the system that may lead to an insecure and unreliable system. Therefore, an accurate method is needed to forecast loads, as is an accurate model that takes into account the factors that affect the growth of the load over a number of years. Furthermore, an accurate algorithm is needed to estimate the parameters of such models.

The growth in electricity consumption in many developing countries has outstripped existing projections, and accordingly, the uncertainties of forecasting have increased [1]. Variables such as economic growth, population, and efficiency standards, coupled with other factors inherent in the mathematical development of forecasting models, make accurate projection difficult $[1,2]$. Unfortunately, an accurate forecast depends on the judgment of the forecaster, and it is impossible to rely strictly on analytical procedures to obtain an accurate forecast.

The objective of the forecasting task is to provide energy and peak-load predictions that meet planning requirements in a consistent and credible manner. A wide variety of techniques for short-term load forecasting (hour-by-hour forecasting) are available in the literature [6-18]; they include the autoregressive moving average (ARMA); Kalman filtering algorithm; artificial neural networks (ANNs) [6-8]; expert system (ES); fuzzy system (FS) [14], etc. A few of them have been applied to longterm annual load forecasting. These techniques range from the simplest approach, such as use of the most recent observation as the forecast, to highly complex approaches, such as an econometric system of simultaneous equations.

The methods used for forecasting electrical peak load and energy for long-term planning fall within two main categories-namely, the econometric and extrapolation methods [3].

Reference [4] applies the least absolute value (LAV) estimation algorithm to estimate the parameters of the annual peak-load model. The model used is a function of the time only (one year is equivalent to one time step). Different orders for the peak-load models are developed. However, all of them are linear in the parameters to be estimated. 
In this chapter we introduce different techniques used to estimate the annual peak load, where different models are used. In the first part, the LAV and least error squares (LES) static state estimation algorithms are used to estimate the parameters of the load model, and a comparative study is performed between the two techniques.

\subsection{Peak-Load-Demand Model}

A model for peak-load demand should take into account the following factors or a part of them, depending on the country in which this model is going to be implemented. There is no unique model that can be applied for utility companies.

These factors are

- The gross domestic product (GDP)

- The population (POP)

- The GDP per capita (GDP/CAP)

- The multiplication of electricity consumption by population (EP)

- The power system losses (LOSS)

- The load factor (LF)

- The cost of one kilowatt-hour (the average rate per unit sale; R/S) (mill/kWh).

The first four factors depend on the behavior of the public; thus, they may vary from country to country, whereas the last three factors depend on the electric power system and the load itself, as well as the consumption of power generated.

Let us begin by putting aside the last three factors for a while, and focus on the first four factors. We call these the country dependency factors. The peak-load demand in this case can be written as

$$
P_{L}=f(\mathrm{GDP})+g(\mathrm{POP})+h(\mathrm{EP})+k(\mathrm{GDP} / \mathrm{CAP})
$$

where $f, g, h$, and $k$ are functions of the variable stated between parentheses. They may be linear and/or nonlinear functions. We assume, for simplicity's sake, linear relations between the peak-load demand and write these factors as

$$
P_{L}=a_{0}+a_{1} \mathrm{GDP}+a_{2} \mathrm{POP}+a_{3} \mathrm{EP}+a_{4}(\mathrm{GDP} / \mathrm{CAP})
$$

where $a_{0}, a_{1}, a_{2}, a_{3}$, and $a_{4}$ are the regression parameters to be determined by the LES and LAV algorithms. The problem now is to determine these parameters using the past data available:

$$
P_{L_{i}}=\left[\begin{array}{lllll}
1 & \mathrm{GDP} & \mathrm{POP} & \mathrm{EP} & \mathrm{GDP} \\
\mathrm{CAP}
\end{array}\right]_{i}\left[\begin{array}{l}
a_{0} \\
a_{1} \\
a_{2} \\
a_{3} \\
a_{4}
\end{array}\right] ; \quad i=1, m
$$


for $i=1, \ldots, m ; m$ is the number of year observations available from past data history; $m \geq 4$. In vector form, equation (9.3) can be rewritten as

$$
Z=H X+\xi
$$

where $Z$ is the $m \times 1$ measurement vector of peak-load demand, $H$ is an $m \times n$ observation matrix containing the factors that affect the peak load, $X$ is the $5 \times 1$ column vector of the load parameters to be estimated, and $\xi$ is the $m \times 1$ error vector to be minimized. At least the past five years' data should be given to determine the peakload-demand parameter $X$.

The solution to equation (9.4) based on the least error squares algorithm is

$$
X^{*}=\left[H^{T} H\right]^{-1} H^{T} Z
$$

Furthermore, the least absolute value algorithm stated in references [4] and [5] is also implemented to compute the best estimate based on LAV minimization criteria. The steps behind the LAV algorithm are explained in reference [3] and Chapter 3.

Having identified the peak-load-demand parameters, we can predict the load for a specified year, using equation (9.1), provided that the other variables in this equation are known in advance for this year.

\subsubsection{Example}

The model proposed in the preceding section is tested using the data for a big utility company [4]; the data are given for the years 1981 to 1988 and listed in Table 9.1. The load model parameters are estimated using only the data of the year 1981 to the year 1988 ( $m=8$ observations). Table 9.2 gives these parameters using the LES and LAV algorithms. Table 9.3 gives the predicted peak-load demand for the years 1989 to 1996 and the percentage error in this prediction using the two estimation algorithms.

The absolute error for both techniques (residual vector) resulting from these parameters for the eight years is given as

$$
\zeta_{\text {LES }}=\left[\begin{array}{r}
-20.6 \\
85.72 \\
-141.21 \\
71.60 \\
68.69 \\
-71.88 \\
-9.69 \\
17.38
\end{array}\right] \quad \text { For the LAV is } \zeta_{\text {LAV }}=\left[\begin{array}{r}
0.00 \\
37.99 \\
-216.87 \\
0.0 \\
0.0 \\
-128.54 \\
0.0 \\
0.0
\end{array}\right]
$$

Note that due to the interpolation property of the LAV, the algorithm fits five data points. The estimated parameters in Table 9.2 are used to predict the peak load for the years from 1989 to 1996. 
Table 9.1 Data for a Big Utility Company (Egyptian Unified Network, or EUN)

\begin{tabular}{|c|c|c|c|c|c|c|c|c|}
\hline Year & $\begin{array}{l}\text { Peak } \\
\text { Load } \\
(\text { GW) }\end{array}$ & $\begin{array}{l}\text { GDP } \\
\text { (Million } \\
\text { EP) }\end{array}$ & $\begin{array}{l}\text { POP } \\
\text { (Million) }\end{array}$ & EP & $\begin{array}{l}\text { GDP/ } \\
\text { CAP }\end{array}$ & $\begin{array}{l}\text { System } \\
\text { Losses } \\
\text { (MW) }\end{array}$ & $\begin{array}{l}\text { Load } \\
\text { Factor } \\
(\%)\end{array}$ & $\begin{array}{l}\text { Cost of } \\
\text { Energy } \\
\text { (Mill/ } \\
\text { kWh) }\end{array}$ \\
\hline 1981 & 3179 & 18985 & 42.11 & 30.11 & 450.85 & 4288.1 & 71.35 & 142.173 \\
\hline 1982 & 3694 & 20628 & 43.33 & 33.58 & 476.07 & 4563.5 & 67.66 & 131.499 \\
\hline 1983 & 3981 & 22450 & 44.50 & 35.67 & 504.49 & 4977.5 & 70.37 & 123.205 \\
\hline 1984 & 4672 & 24042 & 45.77 & 37.06 & 525.26 & 5592.7 & 67.78 & 110.147 \\
\hline 1985 & 5158 & 25691 & 46.99 & 38.60 & 546.73 & 6478.7 & 66.69 & 94.4155 \\
\hline 1986 & 5361 & 26842 & 48.32 & 40.20 & 555.50 & 6159.0 & 68.66 & 101.59 \\
\hline 1987 & 5803 & 27912 & 50.50 & 41.20 & 552.71 & 6862.6 & 69.25 & 86.200 \\
\hline 1988 & 6152 & 29172 & 51.51 & 43.90 & 566.34 & 7479.1 & 70.22 & 70.2631 \\
\hline 1989 & 6279 & 30417 & 52.54 & 46.02 & 578.93 & 7473.9 & 71.96 & 67.2093 \\
\hline 1990 & 6664 & 31726 & 53.59 & 47.96 & 592.00 & 7369.8 & 71.34 & 65.6462 \\
\hline 1991 & 7004 & 32799 & 54.66 & 50.58 & 600.02 & 7411.8 & 70.86 & 63.8323 \\
\hline 1992 & 7215 & 33448 & 55.76 & 52.74 & 599.90 & 8124.7 & 71.96 & 58.9473 \\
\hline 1993 & 7503 & 34282 & 56.87 & 54.58 & 602.80 & 8456.0 & 71.65 & 57.4074 \\
\hline 1994 & 7657 & 35624 & 58.01 & 56.48 & 614.12 & 8415.3 & 72.46 & 58.33 \\
\hline 1995 & 8149 & 37298 & 59.17 & 56.48 & 630.37 & 8555.8 & 71.90 & 61.2318 \\
\hline 1996 & 8491 & 39161 & 60.35 & 58.54 & 648.87 & 8787.4 & 72.23 & 71.8849 \\
\hline
\end{tabular}

Table 9.2 Estimated Parameters for the Peak-Load-Demand Model

\begin{tabular}{lcc}
\hline Parameters & LES Algorithm & LAV Algorithm \\
\hline$a_{0}$ & -2479.2 & -4081.65 \\
$a_{1}$ & 0.329 & 0.2314 \\
$a_{2}$ & 28.5 & 39.39 \\
$a_{3}$ & -37.86 & -10.55 \\
$a_{4}$ & -1.379 & 3.39 \\
\hline
\end{tabular}

The predicted loads as well as the errors in this prediction, using LES and LAV techniques, are given in Table 9.3. Examining this table reveals that both techniques produce fairly good estimates for such type of forecast and such type of peak-load model.

\subsubsection{A More Detailed Model}

Using four variables in the first model may not be adequate; thus, we need an accurate model that takes into account all the factors stated previously. We may assume this model to be 
Table 9.3 Predicted Peak Load and the Percentage Error in This Prediction

\begin{tabular}{|c|c|c|c|c|c|}
\hline \multirow[b]{2}{*}{ Year } & \multirow[t]{2}{*}{ Actual Load } & \multicolumn{2}{|c|}{$\begin{array}{l}\text { LES } \\
\text { Estimates }\end{array}$} & \multicolumn{2}{|c|}{$\begin{array}{l}\text { LAV } \\
\text { Estimates }\end{array}$} \\
\hline & & $\begin{array}{l}\text { Peak-Load } \\
\text { Power }\end{array}$ & $\%$ Error & $\begin{array}{l}\text { Peak-Load } \\
\text { Power }\end{array}$ & $\%$ Error \\
\hline 1989 & 6279 & 6484.72 & -3.26 & 6803.46 & -3.57 \\
\hline 1990 & 6664 & 6853.84 & -2.85 & 6871.56 & -3.11 \\
\hline 1991 & 7004 & 7127.10 & -1.78 & 7161.55 & -5.25 \\
\hline 1992 & 7215 & 7290.35 & -1.04 & 7331.86 & -1.62 \\
\hline 1993 & 7503 & 7522.71 & -0.26 & 7558.99 & -0.75 \\
\hline 1994 & 7657 & 7909.18 & -3.29 & 7932.76 & -3.6 \\
\hline 1995 & 8149 & 8470.57 & -3.95 & 8420.90 & -3.34 \\
\hline 1996 & 8491 & 9013.63 & -6.15 & 8939.46 & -5.28 \\
\hline
\end{tabular}

Table 9.4 Estimated Parameters for a Detailed Peak-Load-Demand Model

\begin{tabular}{llllllllr}
\hline Parameters & \multicolumn{1}{c}{$\boldsymbol{a}_{\mathbf{0}}$} & $\boldsymbol{a}_{\mathbf{1}}$ & \multicolumn{1}{c}{$\boldsymbol{a}_{\mathbf{2}}$} & $\boldsymbol{a}_{\mathbf{3}}$ & $\boldsymbol{a}_{\mathbf{4}}$ & $\boldsymbol{a}_{\mathbf{5}}$ & $\boldsymbol{a}_{\mathbf{6}}$ & \multicolumn{1}{c}{$\boldsymbol{a}_{\mathbf{7}}$} \\
\hline LES & 4713.214 & 0.4192 & -13.38 & -27.997 & -10.389 & 0.1535 & -61.865 & 2.394 \\
LAV & 6398.75 & 0.4932 & -33.835 & -28.328 & -13.8612 & 0.1256 & -73.128 & 4.3864 \\
\hline
\end{tabular}

$$
\begin{aligned}
P_{L}= & a_{0}+a_{1}(\mathrm{GDP})+a_{2}(\mathrm{POP})+a_{3}(\mathrm{EP})+a_{4}(\mathrm{GDP} / \mathrm{CAP}) \\
& +a_{5}(\text { system losses })+a_{6}(\mathrm{LF})+a_{7}(\text { cost of } \mathrm{kWh}) .
\end{aligned}
$$

Equation (9.6) is a linear equation in the parameters to be estimated, $a_{0}$ to $a_{7}$. Thus, equation (9.6) can be rewritten in the form of equation (9.4) as

$$
Z=H X+\xi
$$

In equation (9.6), the following vectors and matrices are defined as follows:

$Z$ is an $m \times 1$ measurement vector of the past history of the peak-load demand;

$H$ is an $m \times 8$ measurement matrix of which the elements contain the seven factors stated in equation (9.6);

$X$ is the $8 \times 1$ load parameters $a_{0}$ to $a_{7}$;

$\xi$ is an $m \times 1$ error vector associated with each measurement to be minimized.

Therefore, we have eight parameters to be estimated, and at least eight measurements should be available to estimate these parameters. Using only eight measurements may produce a poor estimate because we force the errors vector to be zero (because the number of equations equals the number of unknowns). Here, we use 12 measurements to estimate the eight parameters using LES and LAV techniques. The solution to equation (9.7) is similar to that given in equation (9.5). Table 9.4 gives the estimated parameters using both techniques.

The validity of the proposed model and the accuracy of the estimated parameters are checked by implementing the model to predict the peak-load power for the years 
Table 9.5 Predicted Peak-Load Power with the Percentage Errors

\begin{tabular}{llllll}
\hline & & \multicolumn{2}{c}{$\begin{array}{l}\text { LES } \\
\text { Ectual Load }\end{array}$} & \multicolumn{2}{c}{$\begin{array}{l}\text { LAV } \\
\text { Estimates }\end{array}$} \\
\cline { 2 - 6 } & MW & $\begin{array}{l}\text { Peak-Load } \\
\text { Power }\end{array}$ & \% Error & $\begin{array}{l}\text { Peak-Load } \\
\text { Power }\end{array}$ & \% Error \\
\hline 1993 & 7503 & 7535.9 & -0.438 & 7626.93 & -1.652 \\
1994 & 7657 & 7857.9 & -2.624 & 7979.76 & -4.215 \\
1995 & 8149 & 8438.5 & -3.552 & 8611.65 & -5.678 \\
1996 & 8491 & 8994.42 & -5.929 & 9227.78 & -8.68 \\
\hline
\end{tabular}

1993 to 1996, using the factors given in Table 9.1 for the same years. Table 9.5 gives the estimated peak load and the percentage error in these estimates.

Examining Table 9.5 reveals the following observations:

- The predicted load using both techniques is accurate enough for such long-term forecasting.

- The predicted load for this estimation period using eight parameters is almost the same as those using the five parameters stated in Table 9.2.

- The maximum predicted error for LES is about $6 \%$, whereas it is about $9 \%$ for LAV, both for the year 1996. These are fairly good estimates for such long-term forecasting.

\subsubsection{A Time-Dependent Model}

If the year under consideration is taken into account (the time horizon), then the peakload power demand can be written as

$$
\begin{aligned}
P_{L}= & a_{0}+a_{1}(\mathrm{GDP})+a_{2}(\mathrm{POP})+a_{3}(\mathrm{EP})+a_{4}(\mathrm{GDP} / \mathrm{CAP}) \\
& +a_{5}(\text { system losses })+a_{6}(\mathrm{LF})+a_{7}(\text { cost of } \mathrm{kWh})+a_{8}(\text { time })
\end{aligned}
$$

In equation (9.8) the time takes values $0,1, \ldots, T_{f}$, where 0 is the starting year, 1 is the next year, and so on. Furthermore, $T_{f}$ is the number of years minus one used in this study. Equation (9.8) can be rewritten in vector form as

$$
Z=H X+\xi
$$

In equation (9.9), the vectors and matrices are defined as follows:

$Z$ is an $m \times 1$ measurement vector of the past history of the peak-load demand;

$H$ is an $m \times 9$ measurement matrix of which the elements contain the eight factors stated in equation (9.8);

$X$ is the $9 \times 1$ load parameters $a_{0}$ to $a_{8}$;

$\xi$ is an $m \times 1$ error vector associated with each measurement to be minimized.

Therefore, we have nine parameters to be estimated, and at least nine measurements should be available to estimate these parameters. Using nine measurements may produce a poor estimate because we force the errors vector to be zero. Here, we use 12 measurements to estimate the nine parameters using LES and LAV 
Table 9.6 Estimated Parameters for a Time-Dependent Model

\begin{tabular}{lrrrrrrrrr}
\hline Parameters & $\boldsymbol{a}_{\boldsymbol{0}}$ & \multicolumn{1}{c}{$\boldsymbol{a}_{\mathbf{1}}$} & \multicolumn{1}{c}{$\boldsymbol{a}_{\mathbf{2}}$} & \multicolumn{1}{c}{$\boldsymbol{a}_{\mathbf{3}}$} & $\boldsymbol{a}_{\mathbf{4}}$ & \multicolumn{1}{c}{$\boldsymbol{a}_{\mathbf{5}}$} & $\boldsymbol{a}_{\mathbf{6}}$ & $\boldsymbol{a}_{\mathbf{7}}$ & \multicolumn{1}{c}{$\boldsymbol{a}_{\mathbf{8}}$} \\
\hline LES & 5613.5 & -0.0816 & 81.49 & -74.81 & 6.67 & -0.01915 & -62.593 & -4.925 & 397.62 \\
LAV & 5459.73 & 1.133 & -182.43 & 14.473 & -34.8 & 0.3293 & -60.76 & 14.563 & -455.8 \\
\hline
\end{tabular}

Table 9.7 Predicted Peak-Load Power with the Percentage Errors

\begin{tabular}{lllllr}
\hline & & \multicolumn{2}{c}{$\begin{array}{l}\text { LES } \\
\text { Estimates }\end{array}$} & \multicolumn{2}{c}{$\begin{array}{l}\text { LAV } \\
\text { Estimates }\end{array}$} \\
\cline { 2 - 6 } Year & MW & $\begin{array}{l}\text { Peak-Load } \\
\text { Power }\end{array}$ & \% Error & $\begin{array}{l}\text { Peak-Load } \\
\text { Power }\end{array}$ & \% Error \\
\hline 1993 & 7503 & 7553.86 & -0.68 & 7557.13 & -0.455 \\
1994 & 7657 & 7812.18 & -2.027 & 7977.57 & -4.187 \\
1995 & 8149 & 8299.56 & -1.847 & 8763.84 & -7.545 \\
1996 & 8491 & 8541.92 & -0.600 & 9800.82 & -15.428 \\
\hline
\end{tabular}

techniques. The solution to equation (9.9) is similar to that given in equation (9.5). Table 9.6 gives the estimated parameters using both techniques.

The estimated parameters in Table 9.6 are used to predict the peak-load-demand power for the past four years. Table 9.7 gives the predicted load and percentage error in this prediction.

Examining Table 9.7, by using the time horizon, we note that

- The LES algorithm produces an accurate prediction for the peak-load power, whereas the LAV produces a fairly accurate prediction.

- The results obtained using this model, especially for the LES estimation, are better than those mentioned in Table 9.5.

- Examining Tables 9.5 and 9.7, we can conclude that the time horizon has little effect on the prediction of the peak-load power.

\subsection{Time-Series Analysis}

In a time-series analysis model, a time series is constructed that takes into account the effect of load for the previous years on the load for the year in question. The order of this time difference series depends on the accuracy of the prediction needed as well as the data available from the past history. The general form for this time series can be formulated as

$$
P_{L}(k)=a_{1} P_{L}(k-1)+a_{2} P_{L}(k-2)+a_{3} P_{L}(k-3)+\cdots+\cdots+a_{n} P_{L}(k-n)
$$

where $k=K, K-1, K-2, \ldots, 1, K$ is the year in question, and $n$ is the degree of the time series. In this model, we use $n=4$. Equation (9.10) in this case becomes 


$$
P_{L}(k)=\left[\begin{array}{llll}
P_{L}(k-1) & P_{L}(k-2) & P_{L}(k-3) & P_{L}(k-4)
\end{array}\right]\left[\begin{array}{c}
a_{1} \\
a_{2} \\
a_{3} \\
a_{4}
\end{array}\right]
$$

Equation (9.11) can be rewritten in vector form as

$$
P_{L}=B X+\delta
$$

where $P_{L}$ is a $K \times 1$ peak-load power, $B$ is a $K \times 4$ measurement matrix that contains the elements of the previous peak-load power, $X$ is a $4 \times 1$ series parameters vector to be estimated, and $\delta$ is a $K \times 1$ error vector to be minimized.

The estimation problem formulated in equation (9.12) can be solved using the two proposed algorithms, LES and LAV, explained in the previous sections. Having identified the series parameters, we can then predict the peak-load power for the forthcoming year.

\subsubsection{Example for the Time-Series Model}

The time-series model explained in this section is used to predict the load for the utility mentioned in the previous example. First, the series model parameters are estimated using the LES and LAV algorithms. Table 9.8 gives these parameters.

The accuracy of these parameters is tested by predicting the annual peak power from the years 1985 to 1996.

Table 9.9 gives the predicated annual peak-load power and the percentage errors in this prediction using the proposed two algorithms. Examining this table reveals the following:

- The model used in this section is an adequate model.

- Both the LES and LAV techniques produce accurate estimates, but the LES estimates are better than the LAV estimates.

- The results obtained for this model are much better than those obtained in the other proposed models.

- The model in this section is independent of the system variables, but it depends on the history peak-load power available.

\subsubsection{Remarks}

In this section, we discussed the following points:

1. Different models are developed and tested for long-term peak-load power forecasting.

2. We studied the effects of GDP, POP, EP, GDP/CAP, etc. on the performance of each developed model.

Table 9.8 Estimated Parameters for a Time-Series Model

\begin{tabular}{llllr}
\hline Parameters & \multicolumn{1}{c}{$\boldsymbol{a}_{\mathbf{1}}$} & $\boldsymbol{a}_{\mathbf{2}}$ & $\boldsymbol{a}_{\mathbf{3}}$ & \multicolumn{1}{c}{$\boldsymbol{a}_{\mathbf{4}}$} \\
\hline LES & 1.14735 & -0.29612 & 0.78316 & -0.61930 \\
LAV & 1.0020 & -0.081380 & 0.61208 & -0.504513 \\
\hline
\end{tabular}


Table 9.9 Predicted Load Using the Time-Series Model

\begin{tabular}{|c|c|c|c|c|c|}
\hline \multirow[b]{2}{*}{ Year } & \multirow[t]{2}{*}{ Actual Load } & \multicolumn{2}{|c|}{$\begin{array}{l}\text { LES } \\
\text { Estimates }\end{array}$} & \multicolumn{2}{|c|}{$\begin{array}{l}\text { LAV } \\
\text { Estimates }\end{array}$} \\
\hline & & $\begin{array}{l}\text { Peak-Load } \\
\text { Power }\end{array}$ & $\%$ Error & $\begin{array}{l}\text { Peak-Load } \\
\text { Power }\end{array}$ & $\%$ Error \\
\hline 1985 & 5158 & 5106 & 1.00 & 5014 & 2.8 \\
\hline 1986 & 5361 & 5305 & 1.10 & 5217 & 2.6 \\
\hline 1987 & 5803 & 5768 & 0.60 & 5671 & 2.28 \\
\hline 1988 & 6152 & 6152 & 0.00 & 5969 & 2.97 \\
\hline 1989 & 6279 & 6343 & -1.0 & 6183 & -1.02 \\
\hline 1990 & 6664 & 6688 & -0.40 & 6548 & 1.74 \\
\hline 1991 & 7004 & 7041 & -0.50 & 6851 & 2.2 \\
\hline 1992 & 7215 & 7256 & -0.57 & 7104 & -0.57 \\
\hline 1993 & 7503 & 7549 & -0.60 & 7450 & -0.61 \\
\hline 1994 & 7657 & 7885 & -3.00 & 7776 & -2.98 \\
\hline 1995 & 8149 & 8134 & 0.20 & 8077 & 0.88 \\
\hline 1996 & 8491 & 8416 & 0.90 & 8436 & 0.65 \\
\hline
\end{tabular}

3. We studied the applications of two parameter estimation algorithms, the LES and LAV algorithms, on the prediction of the annual peak load.

4. In the time-dependent model, the LES algorithm produces better-predicted results than the LAV algorithm.

5. The time-series model is the best one for such systems because it has the lowest error rate among the other developed models.

It is worthwhile to state here that every power system has its own model; the one suitable for a particular system may not be suitable for another system.

\subsection{Kalman Filtering Algorithm}

Long-term forecasting is characterized by its high uncertainty owing to its high dependence on socioeconomic factors; for this reason, an error level up to $10 \%$ is acceptable [28]. These results are highly dependent on uncertain parameters such as electric utility, region, country, economic growth, population growth, and population habits. Moreover, the data on which the long-term forecasting technique is tested play an important factor in determining the level of the forecast error. An algorithm that gives a low average forecast error for a certain electric utility in a certain country may not give the same level of error for a different utility in a different country. Therefore, any attempt for comparing different forecasting techniques should utilize the same testing data.

The technique used in this section combines regression estimation with a timeseries load model suited for the Kalman filtering approach. Historic load data over 
a certain period of time, say one year, are arranged in a two-dimensional (2D) (24 hours $\times$ 52 weeks) layout. It is worth mentioning that a time period of one year is highly suggested not only because this period provides a reasonable amount of data, but also because it entirely exploits the underlying daily and seasonal load variations. The technique used in this section employs the following primary features of the long-term forecasting problem:

- Seasonal and daily load-demand behavior: The cyclic behavior of the load demand in response to seasonal and daily variations is modeled using short-term linear regression techniques over a specific period of time (one year). The short-term forecasting accuracy is high due to the high correlation of the load time series. Therefore, the resulting model is reasonably accurate and establishes the basis for future (next year) trends of the load demand.

- Annual load-demand growth: The overall load demand of a system continually increases due to population and industrial growth as well as increases in industrial consumption. A third-order regression model is used to develop the annual growth in load demand as a function of time. The annual growth provides an approximate correction factor for the load-demand behavior for the next year.

Any long-term forecast is always inaccurate due to the complexity of the loadaffecting factors. For example, peak demand is very much dependent on temperature. The fluctuation in temperature is extremely hard to forecast for a long period of time. Therefore, the main objective of long-term load forecasting is to increase forecast accuracy. The load time-series behavior is developed as a linear time-varying mathematical model relating the load at time instant $k$ as a function of the load at time instances less than $k$. The load model is then used to form a time-varying discrete dynamic system suited for the Kalman filter, which is employed to estimate and predict the next year's load demand. The Kalman filter is fed with the estimated load augmented with the annual load growth obtained from the previous two steps as measurement values.

\subsubsection{Estimating Multiple Regression Models}

The electric load depends on a number of complex factors that have nonlinear characteristics, and good results may not be obtained using a single linear model. The approach taken in this section is the decomposition of the problem into multiple simple (first-order) linear regression models to capture the global nonlinear behavior of the load. Each of the linear regression models extracts the short-term correlation of a certain set of data. One year's data are arranged into a two-dimensional layout with 24 columns representing 24 hours of a day and 52 rows representing 52 weeks of the year. Figure 9.1 illustrates the 2D layout of the load data.

Special consideration is taken for the load variation during the weekends. Accordingly, weekends are treated separately but in exactly the same manner as the working days. The $L(i, k)$ cell in Figure 9.1 is the average load of the working days of the $i$ th week at the $k$ th hour. With this setup of the load data, obvious great intrinsic correlations exist between successive columns as well as between successive rows, as illustrated in Figures 9.2 and 9.3, respectively. These two figures, and all subsequent results, are based on the load demand of one of the largest electric power utilities in Canada for the years 1994 and 1995. 


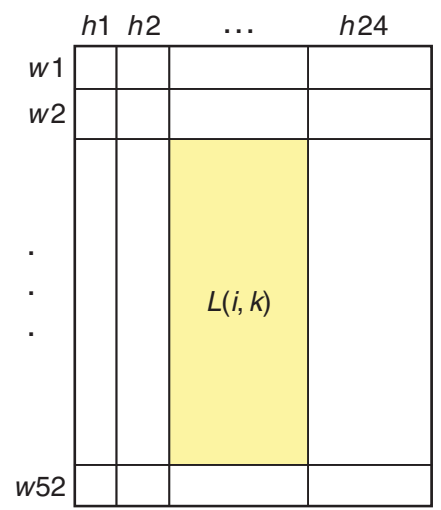

Figure 9.1 Two-dimensional layout of the load data.

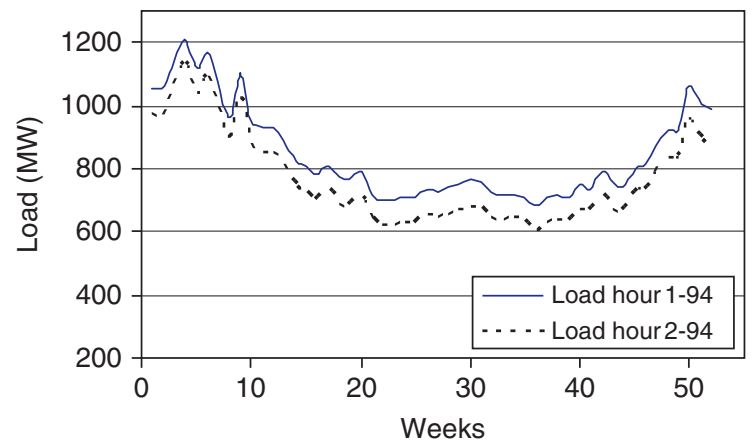

Figure 9.2 Comparing weekly average load of hour 1 and hour 2, 1994.

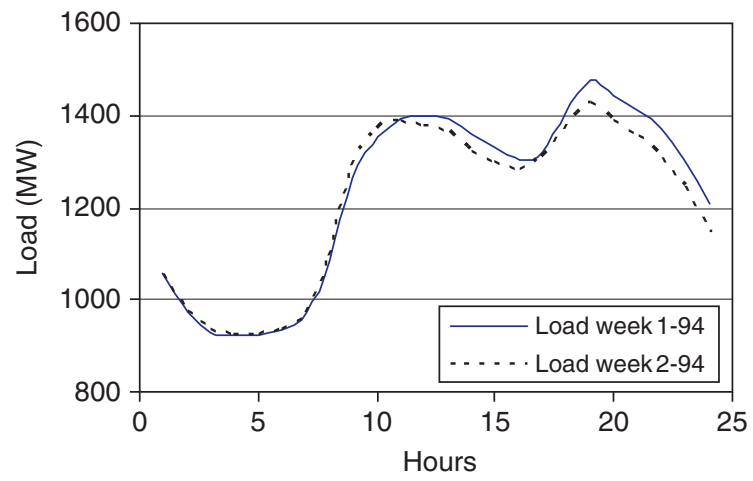

Figure 9.3 Comparing weekly average load of weeks 1 and 2, 1994. 
Figure 9.2 shows the load correlation between hour 1 AM and hour 2 AM throughout the whole year 1994. The correlation factor was calculated as 0.997. Similarly, Figure 9.3 shows the load correlation of week 1 and week 2 of year 1994, with a correlation factor of 0.985 . The strong correlation is maintained over the entire year for all 24 hours of the day, as illustrated in Figures 9.4 and 9.5.

The persistent correlation of the prevalent load patterns suggests the use of shortterm simple linear regression models for successive hours (see equation (9.13a)) and another set for successive weeks (see equation (9.13b)). This results in $24 \times 52$ simple linear regression models, which are used to draw the shape of the 2D load behavior contour for one year.

$$
\begin{aligned}
& L(i, k)=a(k) L(i, k-1)+b(k) \quad k=1, \ldots, 24 \\
& L(i, k)=c(i) L(i-1, k)+d(i) \quad i=1, \ldots, 52
\end{aligned}
$$

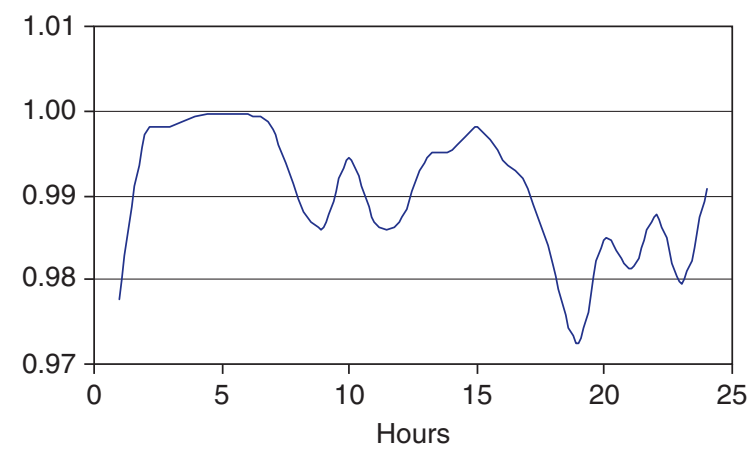

Figure 9.4 Correlation factor for successive hours of 52 weeks of 1994.

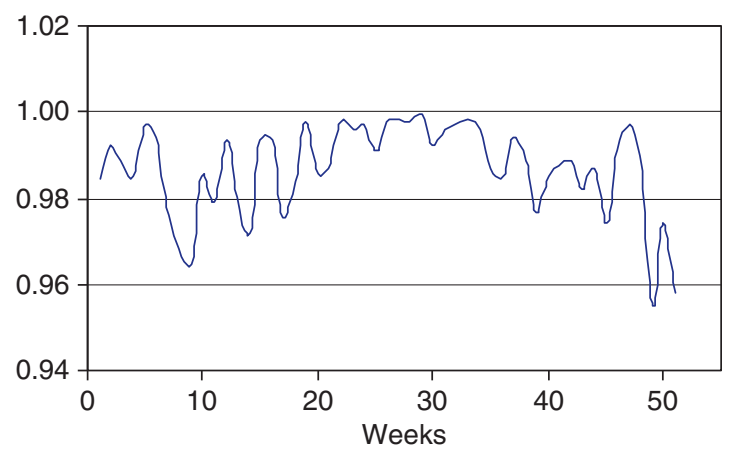

Figure 9.5 Correlation factor for successive weeks over 24 hours of 1994. 
where

$a(k)$ and $b(k)$ are regression parameters at the $k$ th hour; $k=1,2, \ldots, 24$, which are determined using the load pairs $[L(i, k), L(i, k-1)]$ for all $i=1,2, \ldots, 52$, by the least squares method; $L(i, k)$ and $L(i, k-1)$ are the weekly average load at hours $k$ and $k-1$, respectively, for all weeks $i=1, \ldots, 52$, with the initial condition $L(i, 0)=L(i-1,24)$;

$c(i)$ and $d(i)$ are regression parameters of the $i$ th week, $i=1,2, \ldots, 52$, which are determined using the load pairs $[L(i, k), L(i-1, k)]$ for all $k=1,2, \ldots, 24$, by the least squares method; $L(i, k)$ and $L(i-1, k)$ are the weekly average load in the $i$ th and $(i-1)$ th weeks, respectively, for all hours $k=1, \ldots, 24$, with the initial condition $L(0, k)=[L(52, k)$ of the previous year].

\subsubsection{Estimating the Next Year's Load Contour}

The preceding regression models are used to project the load trends for the next year. Figures 9.6 and 9.7 demonstrate the fact that successive years have nearly identical load behavior contours.

A recursive procedure used to estimate next year's load contour utilizing regression models of the previous year is as follows:

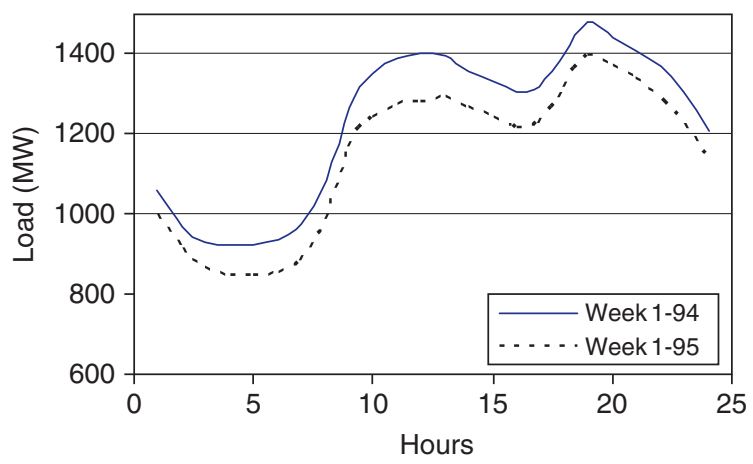

Figure 9.6 Comparing average weekly loads for various weeks of 1994 and 1995 .

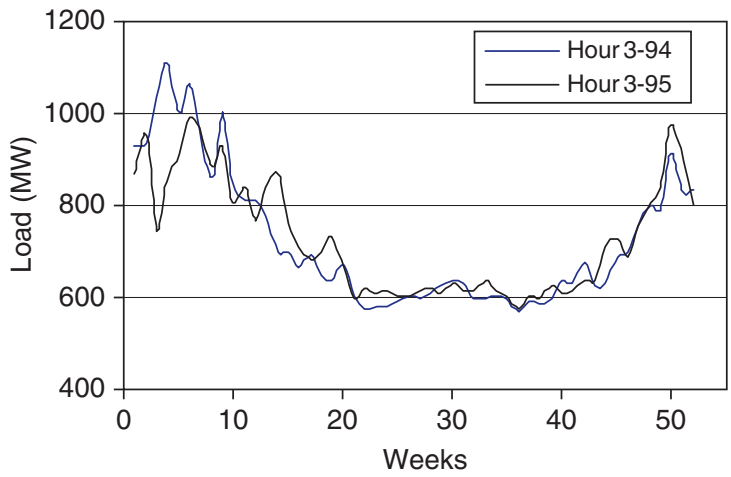

Figure 9.7 Comparing average weekly loads for various hours of 1994 and 1995. 
1. Estimate for the first week the weekly average load: This process corresponds to estimating the first row of the next year's load; refer to Figure 9.8(a). Using equation (9.13b), we calculate $\hat{L}(1, k)$

$$
\hat{L}(1, k)=c(k) \hat{L}(0, k)+d(k) \quad k=1,2, \ldots, 24
$$

where $\hat{L}(1, k)$ is the estimated weekly average load of the first week at the $k$ th hour; $\hat{L}(0, k)$

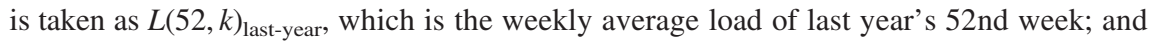
$[c(k), d(k)]$ is a pair of regression coefficients of the $k$ th hour, obtained from equation (9.13b) using last year's data.

2. Estimating for the first hour the weekly average load: This process corresponds to estimating the first column of the next year's load; refer to Figure 9.8(b). Using equation (9.13a), we calculate $\hat{L}(i, 1)$

$$
\hat{L}(i, 1)=a(i) \hat{L}(i, 0)+b(i) \quad i=2,3, \ldots, 52
$$

where $\hat{L}(i, 1)$ is the estimated weekly average load of the first hour in the $i$ th week; $\hat{L}(i, 0)$ is taken as $L(i-1,24)$, which is the weekly average load of the 24 th hour of the previous week; and the $[a(i), b(i)]$ is a pair of regression coefficients of the $i$ th week, obtained from equation (9.13a) using last year's data.

3. Estimating for the second week the weekly average load: This process corresponds to estimating the second row of the next year's load; refer to Figure 9.8(c). Using equation (9.13b), we calculate $\hat{L}(2, k)$

$$
\hat{L}(2, k)=c(k) \hat{L}(1, k)+d(k) \quad k=2,3, \ldots, 24
$$

where $\hat{L}(2, k)$ is the estimated weekly average load of the second week at the $k$ th hour; and $\hat{L}(1, k)$ is obtained using equation (9.14).

4. Estimating for the second hour the weekly average load: This process corresponds to estimating the second column of the next year's load; refer to Figure 9.8(d). Using equation (9.13a), we calculate $\hat{L}(i, 2)$

$$
\hat{L}(i, 2)=a(i) \hat{L}(i, 1)+b(i) \quad i=3,4, \ldots, 52
$$

where $\hat{L}(i, 2)$ is the estimated weekly average load of the second hour in the $i$ th week; and $\hat{L}(i, 1)$ is obtained using equation $(9.15)$.

5. The recursive iterations are repeated until $i=52$ and $k=24$.

6. Steps 1 through 5 are repeated for forecasting more years.

The preceding procedure produces a two-dimensional contour of the load behavior for one year based on regression coefficients of the previous year. The load contour will then be augmented by the annual load growth to account for the load change between successive years.

\subsection{Annual Load Growth}

To maximize the accuracy of next year's load-demand estimation, we estimate and employ annual load growth as an adjusting factor. It is evident that there is a very strong 


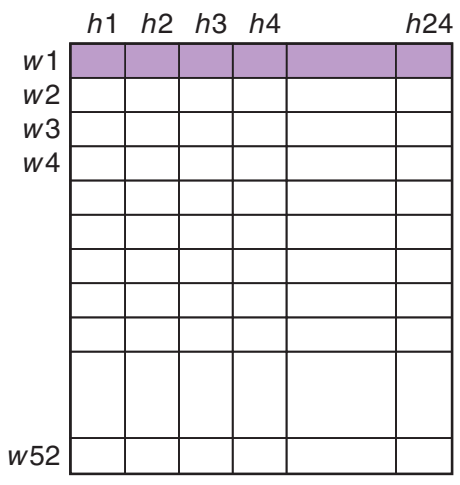

(a)

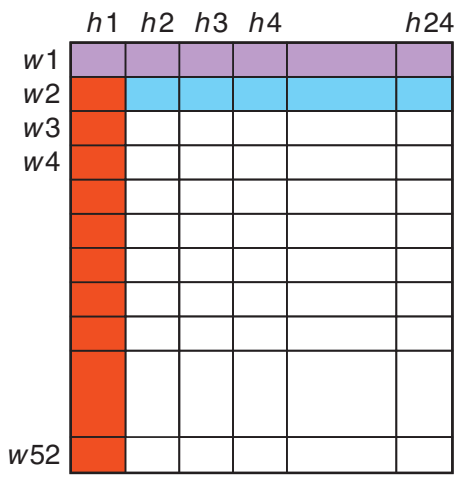

(c)

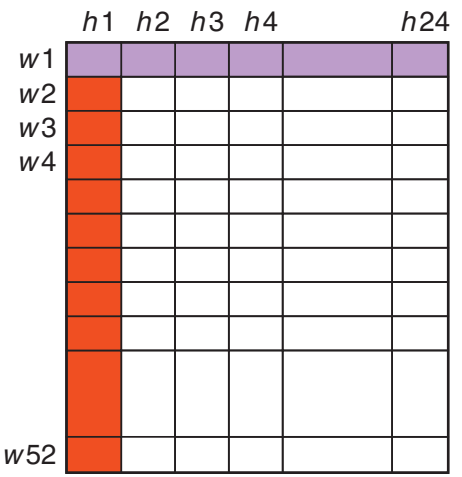

(b)

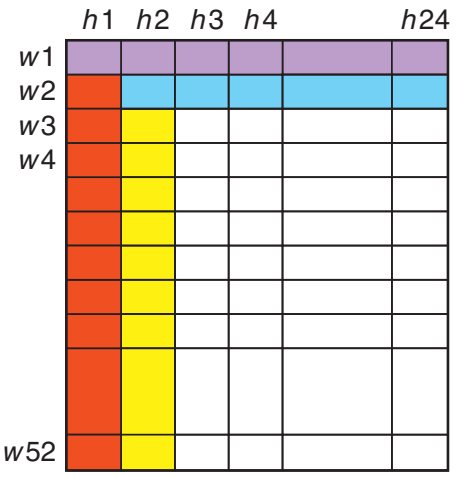

(d)

Figure 9.8 (a) First week (row) load estimation resulting from the first iteration. (b) First hour (column) load estimation resulting from the second iteration. (c) Second week (row) load estimation resulting from the third iteration. (d) Second hour (column) load estimation resulting from the fourth iteration.

dependence of the load demand on time. Typical load profiles of successive years reveal very strong correlation at certain periodic time intervals. For example, refer to Figure 9.7; the two load curves at a certain hour over the whole year for two successive years retain the same shape. Moreover, there is, on average, a clear load increase over the previous year. This increase amounts to an annual load growth at that hour as a function of time (weeks) throughout the whole year. The load growth is modeled as the difference between the load curves of two successive years as a function of time.

A third-order polynomial is utilized to model the load as a function of time at the $k$ th hour as a function of the load of the previous hour. The regression model is as follows:

$$
L(i, k)=\beta_{0}(k)+\beta_{1}(k) L(i, k-1)+\beta_{2}(k) L^{2}(i, k-1)+\beta_{3}(k) L^{3}(i, k-1)
$$




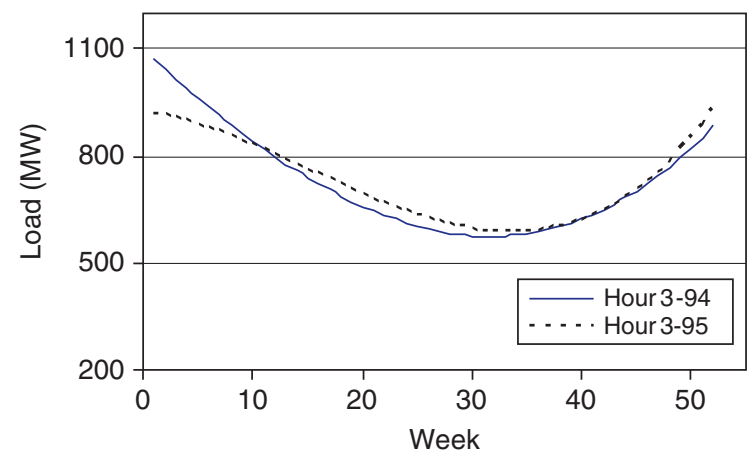

Figure 9.9 Approximate curves of load of hour 3 of 1994 and 1995.

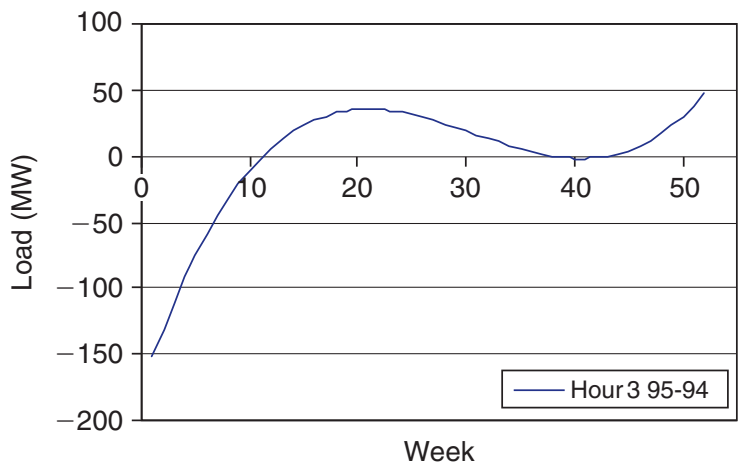

Figure 9.10 Annual load growth variations during 52 weeks of a year.

where $\beta_{j}(k), j=0,1,2,3$ are regression variables at the $k$ th hour, and $k=1,2, \ldots, 24$, which are determined using the load pairs $[L(i, k), L(i, k-1)$ for all $i=1,2, \ldots, 52]$ by the least squares method. The initial values $L(i, 0)$ are set to $L(i-1,24)$. The two curves that approximate the relationship between $L(i, k)$ and $L(i, k-1)$ corresponding to the load behavior of the two years in Figure 9.7 are shown in Figure 9.9.

Next, the procedure for evaluating the annual load growth is as follows; we assume that the annual load growth is calculated between 1993 and 1994 to be used for predicting the 1995 load:

1. Using equation (9.18), we determine the regression coefficients ( 24 sets) for 24 hours for the year 1993. The coefficients define 24 approximate curves of the weekly average load, one curve per hour.

2. We repeat the calculations of the previous step to the 1994 data.

3. We define the annual load growth as the difference of the approximate load curves of 1994 and 1993:

$$
\text { Annual Load Growth }(i)=L(i, k)_{(95)}-L(i, k)_{(94)} \quad k=1,2, \ldots, 24, \quad i=1,2, \ldots, 52
$$

The annual load growth curve is obtained by subtracting the approximate curve of 1994 from the approximate curve of 1993, as shown in Figure 9.10. 
After estimating the shape of the load contour and augmenting it with the annual load growth, we use the Kalman filtering algorithm to predict the next year's load demand. First, we express the dynamic variation of load with respect to load values at previous hours as a time-varying linear model. Second, we construct a dynamic time-varying state space model and adapt it for the Kalman filtering technique. Last, we use the estimated regression and the annual load growth results as measurement inputs for the Kalman filtering algorithm.

\subsubsection{Load Modeling for the Kalman Filtering Algorithm}

Generally, the load at any discrete time instant $k=1,2, \ldots, 24$, corresponding to 24 hours of one day, can be expressed as a fourth-order time-varying linear model as follows:

$$
L(i, k)=\alpha_{0}(k)+\alpha_{1}(k) L(i, k-1)+\alpha_{2}(k) L(i-1, k)+\alpha_{3}(k) L(i-1, k-1)
$$

where

$L(i, k)=$ weekly average load at time instant: $i$ th week and $k$ th hour;

$\alpha_{0}(k)=$ base load at time instant $k$;

$\alpha_{j}(k)=j=1,2,3$, load coefficients at the $k$ th hour.

The model assumes that the load coefficients are constant over each discrete time instant $k=1, \ldots, 24$, of the 24 hours of the day. Parameter estimation is carried out for each of the 24 discrete instances in a day. Accordingly, 24 sets of coefficients are required to be estimated for one day. The estimated coefficients can be plugged into the model to predict hourly loads for the next day.

\subsubsection{Kalman Filter Parameter Estimation Algorithm}

In this section we address only the necessary equation for the development of the basic recursive discrete Kalman filter. Given the discrete state equations

$$
\begin{aligned}
& x(k+1)=A(k) x(k)+w(k) \\
& z(k)=C(k) x(k)+v(k)
\end{aligned}
$$

where

$x(k)$ is $n \times 1$ system states;

$A(k)$ is an $n \times n$ time-varying state transition matrix;

$z(k)$ is an $m \times 1$ measurement vector;

$C(k)$ is an $m \times n$ time-varying output matrix;

$w(k)$ is an $n \times 1$ system error;

$v(k)$ is an $m \times 1$ measurement error.

The noise vectors $w(k)$ and $v(k)$ are uncorrelated white noises. The basic discrete-time Kalman filter algorithm recursive equations appropriate for forecasting problems were 
discussed earlier. The load model is used to form a time-varying discrete dynamic system relevant to the Kalman filter. The dynamic system of equation (9.21) is used with the following definitions:

1. The state transition matrix, $A(k)$, is a constant $4 \times 4$ identity matrix.

2. The error covariance matrices are chosen to be identity matrices for this simulation; they would be assigned better values if more knowledge were obtained on the sensor accuracy and process error.

3. The state vector, $x(k)$, consists of four parameters: $\left[\alpha_{0}(k), \alpha_{1}(k), \alpha_{2}(k), \alpha_{3}(k)\right]^{T}$.

4. $C(k)$ is a four-element time-varying row vector, which relates the measured load data to the state vector. (Refer to equation (9.21).)

5. The observation vector, $z(k)$, for this application is a scalar representing the load at time instant $k$. (Refer to equation (9.21).)

The observation equation $z(k)=C(k) x(k)$ has the form

$$
z(k)=\hat{L}(i, k)=\left[\begin{array}{llll}
1 & \hat{L}(i, k-1) & \hat{L}(i-1, k) & \hat{L}(i-1, k-1)
\end{array}\right]\left[\begin{array}{c}
\alpha_{0}(k) \\
\alpha_{1}(k) \\
\alpha_{2}(k) \\
\alpha_{3}(k)
\end{array}\right]
$$

where the parameters and load values are defined in equation (9.20), with $k$ representing the time instant of the 24 discrete hours of the day, $k=1, \ldots, 24 . \hat{L}(i, k)$ is the estimated weekly average load using regression parameters and annual load growth. For any time instant $k$, the Kalman filter iterates over all available load data, $\hat{L}(i, k)$ for all weeks, $i=1, \ldots, 52$, with additional interpolation load points to estimate the set of parameters $\left[\alpha_{0}(k), \alpha_{1}(k), \alpha_{2}(k), \alpha_{3}(k)\right]$. Interpolation of load points is required to accelerate Kalman filter convergence by increasing its input data.

\subsection{Computer Exercises}

To verify the effectiveness of the proposed load-demand forecasting technique, we used load data for one of the largest utility companies in Canada for the years 1994 and 1995. Regression models are obtained from 1994 data and used to project load demand for 1995. Kalman filtering is used to increase the estimation accuracy of the year 1995, and then the forecasted results are compared with the actual data of 1995 .

\subsubsection{Multiple Regression Models Results}

Using equation (9.13a), we calculate 24 sets of regression coefficients. Table 9.10 shows the first seven of these sets as a sample. This table also lists the correlation factors of successive hours (columns) of the 1994 load data. Similarly, using equation (9.13b), we calculate 52 sets of regression coefficients. Table 9.11 shows the first seven of these sets as a sample, together with the correlation factors of successive weeks (rows) of the 1994 load data. 
Table 9.10 Correlation Factors and Regression Coefficients for Seven Hours of 1994

\begin{tabular}{|c|c|c|c|c|c|c|c|}
\hline 1994 & Hour 1 & Hour 2 & Hour 3 & Hour 4 & Hour 5 & Hour 6 & Hour 7 \\
\hline $\begin{array}{l}k=\text { hour of } \\
\text { the day }\end{array}$ & 1 & 2 & 3 & 4 & 5 & 6 & 7 \\
\hline \multicolumn{8}{|l|}{ Correlation } \\
\hline Factor & 0.978 & 0.997 & 0.998 & 0.999 & 0.999 & 1.000 & 0.998 \\
\hline$a(k)$ & 0.973 & 0.994 & 1.014 & 1.022 & 1.025 & 1.024 & 1.049 \\
\hline$b(k)$ & -89.311 & -76.835 & -49.053 & -31.009 & -21.580 & -11.659 & -6.003 \\
\hline
\end{tabular}

Table 9.11 Correlation Factors and Regression Coefficients for Seven Weeks of 1994

\begin{tabular}{llllr}
\hline 1994 & $\begin{array}{l}\boldsymbol{i}=\text { Week } \\
\text { Number }\end{array}$ & $\begin{array}{l}\text { Correlation } \\
\text { Factor }\end{array}$ & $\boldsymbol{c}_{(\boldsymbol{i})}$ & \multicolumn{1}{c}{$\boldsymbol{d}_{(\boldsymbol{i})}$} \\
\hline Week 1 & 1 & 0.985 & 0.918 & 80.911 \\
Week 2 & 2 & 0.993 & 0.964 & 137.674 \\
Week 3 & 3 & 0.987 & 0.953 & -86.209 \\
Week 4 & 4 & 0.985 & 0.983 & 43.987 \\
Week 5 & 5 & 0.997 & 1.025 & -5.718 \\
Week 6 & 6 & 0.994 & 0.909 & -252.143 \\
Week 7 & 7 & 0.976 & 1.161 & \\
\hline
\end{tabular}

\subsubsection{Estimating the 1995 Load Contour}

The mean absolute percentage error (MAPE) with respect to the actual load is used to measure the effectiveness of the estimated results. For $n$ estimated load values, the MAPE error is given by the equation

$$
\text { MAPE }=\frac{100}{n} \sum_{i=1}^{n} \frac{\left|\hat{L}_{e s t, i}-L_{a c t, i}\right|}{L_{a c t, i}}
$$

where $\hat{L}_{e s t, i}$ and $L_{a c t, i}$ are the estimated and actual $i$ th load values, respectively.

The recursive procedure outlined in Section 9.4.2 is used to project the shape of the 1995 load contour. The regression coefficients determined earlier-namely $[c(i), d(i)]$ and $[a(k), b(k)]$ - are alternatively used to estimate a row and a column, respectively, of the 1995 contour described in Figure 9.1. The procedure is carried out for 24 iterations converging to the actual 1995 load. Figure 9.11 shows a sample of the MAPE error convergence for each hour over the 24 iterations. As shown, the error for each hour converges to its minimum. The overall MAPE error for the whole year was found to be $5.12 \%$. 


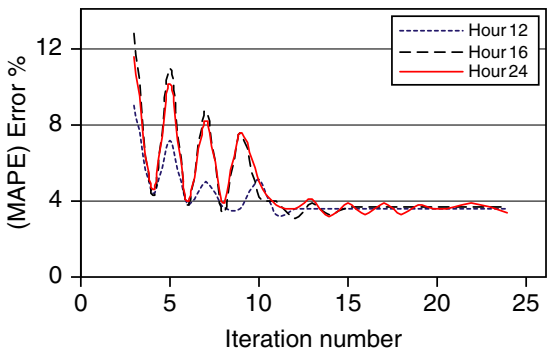

(a)

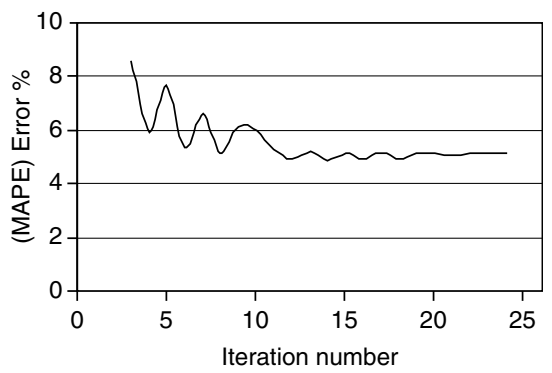

(b)

Figure 9.11 (a) Regression estimation (MAPE) error over 52 weeks of 1995; (b) overall regression estimation (MAPE) error over 52 weeks of 1995.

\subsubsection{Kalman Filter Prediction Results}

To prepare the input (measurement) data for Kalman filtering, we produce the annual load growth and use it to augment the estimated load contours determined in Section 9.7.2. The third-order polynomial load models described in equation (9.18) are used to calculate the annual load growth for each hour of the day. Figure 9.9 shows the approximate fitted curves for hour 3 of 1994 and 1995, and Figure 9.10 shows the annual load growth for that hour. The annual load growth curves for all hours follow almost the same shape with very minimal variations, as illustrated by Figure 9.12. During almost the first 10 weeks, the annual load growth is negative. This accounts for the unexpectedly low load demand during these weeks in 1995, as noticed in Figure 9.7. The low power consumption in these weeks of 1995 was mainly due to the above-normal high temperatures. The model naturally responds to the given data. It will react differently to different data from different utilities. To reduce the dependency of the annual load growth on uncontrollable short-term weather variations, we can calculate the average of the annual growth over several years. Furthermore, second-order models will not be sufficient to pick up such annual load growth variations. Third-order models or higher must be used. Models with orders $3,4,5$, and 6 were tested. It was found that models with orders higher than third order were very sensitive to round-off errors and produce "very" incorrect results.

The fourth-order dynamic time-varying state space model for the Kalman filter described in Section 9.5.2 is employed to implement the following steps:

Step 1 The initial condition of the parameter vector is fixed arbitrarily to ones.

Step 2 Run the Kalman filter for the first hour of the day (the first column of Figure 9.1) using the actual load values of 1994 in the observation equation, equation (9.22), of the Kalman filter model. We used Cubic-Hermit interpolation to generate five extra points between each pair of load values to boost up the Kalman filter convergence. Save the four load-model estimated parameters for prediction. Set $i=1$ ( $i$ represents the week number of 1995).

Step 3 Predict the load value of the $i$ th week of 1995 using the saved load-model estimated parameters:

Set $i=i+1 ;$ if $i$ is greater than 52 weeks, go to step 5 . 


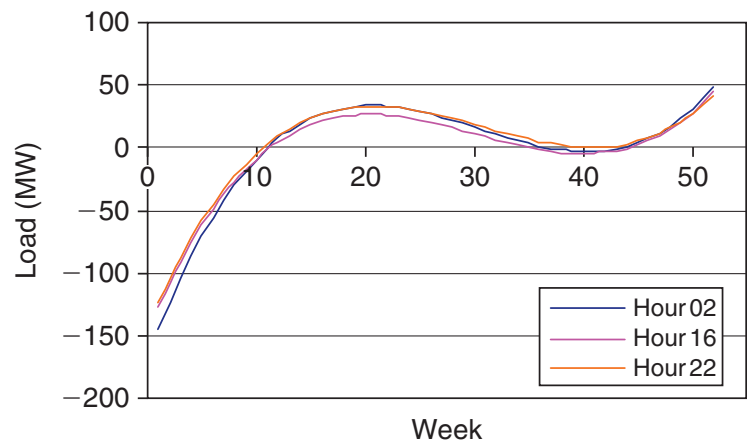

Figure 9.12 Annual load growth throughout 52 weeks of the year.

Step 4 Use estimated regression load values of the $i$ th week of 1995 data (from Section 5.2) in the measurement equation, equation (9.22), to estimate the next set of load-model parameters using the Kalman filter. Save the load-model parameters. Go to step 3.

Step 5 Use the estimated parameters of the previous hour as the initial condition for estimating the next hour's coefficient using the Kalman filter. Repeat steps 3 and 4 for all 24 hours of the day.

The five steps of the preceding algorithm are illustrated using the flow diagram in Figure 9.13.

Figure 9.14 presents the estimated Kalman filter load-model parameters. As illustrated, all estimated parameters converge to their steady-state value after some transient fluctuations. Table 9.12 presents only a sample (10 weeks) of the estimated load using the Kalman filter together with the actual load demand of 1995. The large MAPE error in some weeks, especially in the second to fourth weeks, is attributed to the sudden, unexpected, and aberrant load condition in either year, which could not be explained by any model variables. The prediction method used captures the general behavior of the load over the year based on the previous year's load data rather than its short-term fluctuations. To reduce such aberrant effects, we could base the load prediction on the average of several previous years instead of only one year's data. Figure 9.15 illustrates the improvement in accuracy of the Kalman filtering algorithm by reducing the error compared to that obtained by the regression technique. A comparison between loads resulting from the estimated parameters and the actual load is shown in Figures 9.16 and 9.17. The results show how closely the estimated model matches with the actual load. Figure 9.16 also displays the MAPE error between the estimated and actual loads. The overall MAPE error for the whole year 1995 was calculated to be $2.24 \%$, and the overall standard deviation was found to be $4.6 \mathrm{MW}$.

\subsubsection{Remarks}

This part of the chapter presented a composite technique for long-term load forecasting using multiple linear regression models and the Kalman filtering algorithm. Simple linear regression models, which capture 2D load behavior over one year, are utilized 


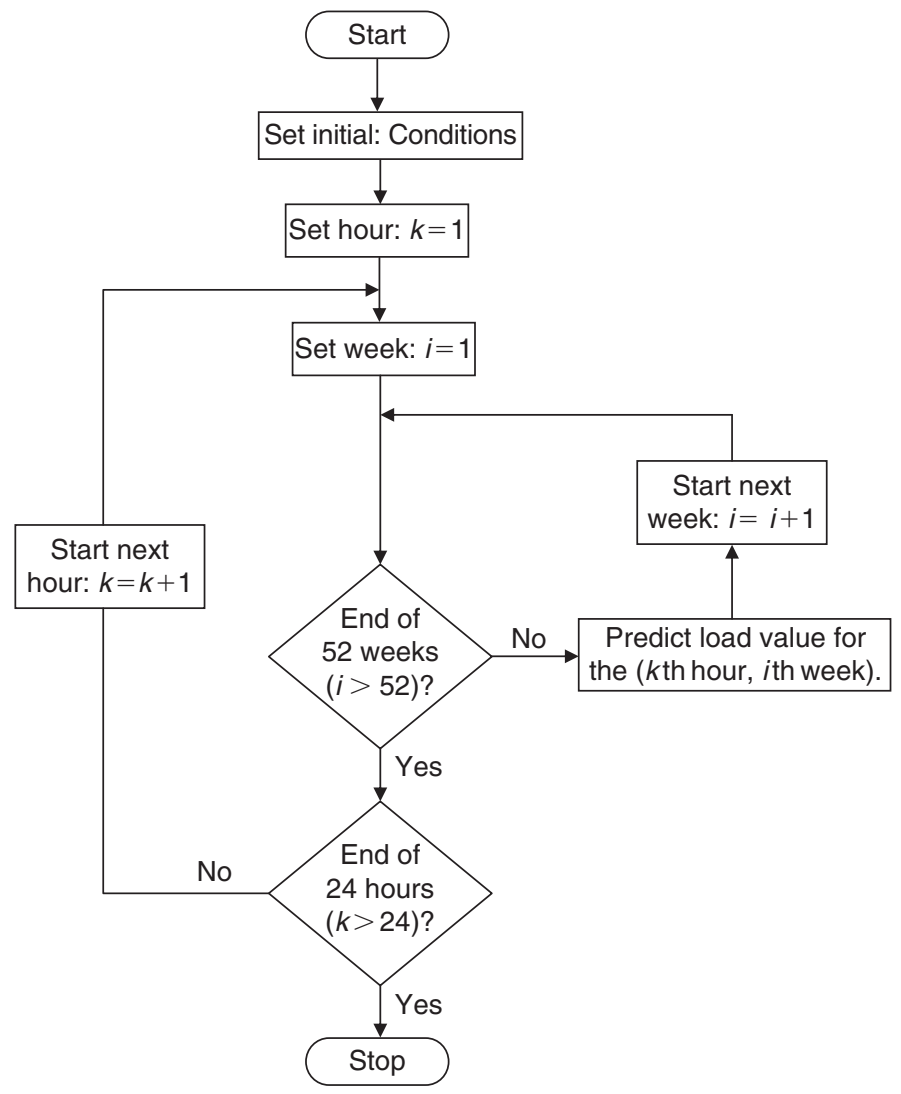

Figure 9.13 Kalman filtering load prediction flow diagram.
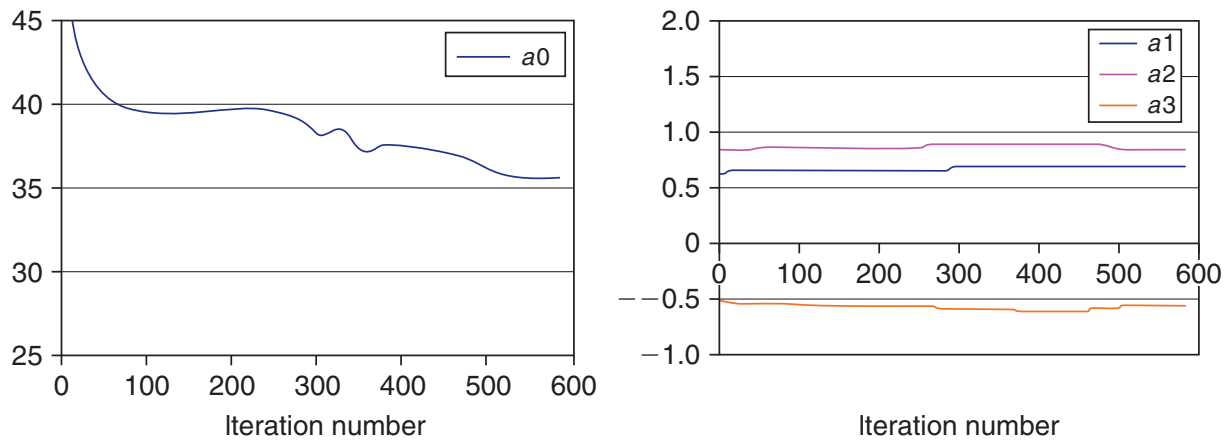

Figure 9.14 Kalman filter load-model parameters' convergence, hour 9. 
Table 9.12 Sample of the Predicted Weekly Average Load Results for 1995 Using the Kalman Filter

\begin{tabular}{|c|c|c|c|c|c|c|c|c|c|c|c|c|}
\hline \multirow[b]{2}{*}{ Week } & \multicolumn{3}{|c|}{ Hour 1} & \multicolumn{3}{|c|}{ Hour 7} & \multicolumn{3}{|c|}{ Hour 17} & \multicolumn{3}{|c|}{ Hour 22} \\
\hline & Actual & Predicted & MAPE & Actual & Predicted & MAPE & Actual & Predicted & MAPE & Actual & Predicted & MAPE \\
\hline 1 & 998 & 993 & 0.57 & 887 & 871 & 1.76 & 1228 & 1212 & 1.36 & 1289 & 1277 & 0.89 \\
\hline 2 & 1078 & 1000 & 7.25 & 1002 & 893 & 10.82 & 1293 & 1206 & 6.75 & 1339 & 1274 & 4.87 \\
\hline 3 & 871 & 1100 & 26.40 & 767 & 1010 & 31.69 & 1136 & 1286 & 13.27 & 1145 & 1368 & 19.51 \\
\hline 4 & 967 & 1151 & 19.09 & 880 & 1056 & 20.01 & 1244 & 1325 & 6.45 & 1302 & 1399 & 7.43 \\
\hline 5 & 1024 & 1056 & 3.10 & 955 & 982 & 2.84 & 1238 & 1265 & 2.15 & 1304 & 1342 & 2.87 \\
\hline 6 & 1101 & 1115 & 1.22 & 1055 & 1025 & 2.82 & 1290 & 1313 & 1.80 & 1375 & 1385 & 0.71 \\
\hline 7 & 1082 & 1007 & 6.93 & 1017 & 928 & 8.72 & 1200 & 1219 & 1.59 & 1298 & 1287 & 0.81 \\
\hline 8 & 999 & 939 & 5.93 & 924 & 872 & 5.60 & 1213 & 1190 & 1.91 & 1265 & 1268 & 0.27 \\
\hline 9 & 1040 & 1043 & 0.26 & 976 & 961 & 1.51 & 1253 & 1244 & 0.70 & 1307 & 1322 & 1.21 \\
\hline 10 & 924 & 914 & 1.13 & 843 & 835 & 0.95 & 1179 & 1160 & 1.61 & 1222 & 1237 & 1.18 \\
\hline
\end{tabular}




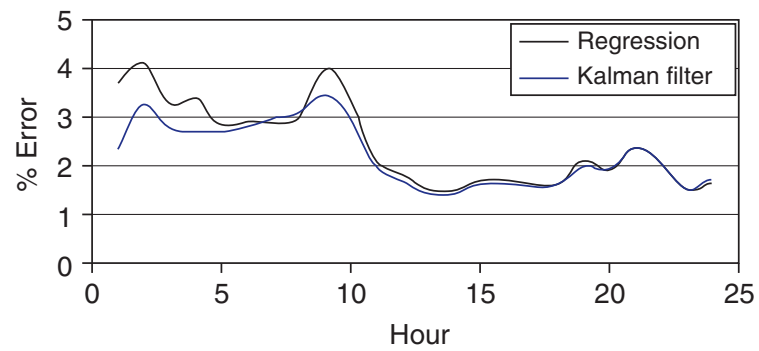

Figure 9.15 Comparing regression and Kalman filter MAPE errors.
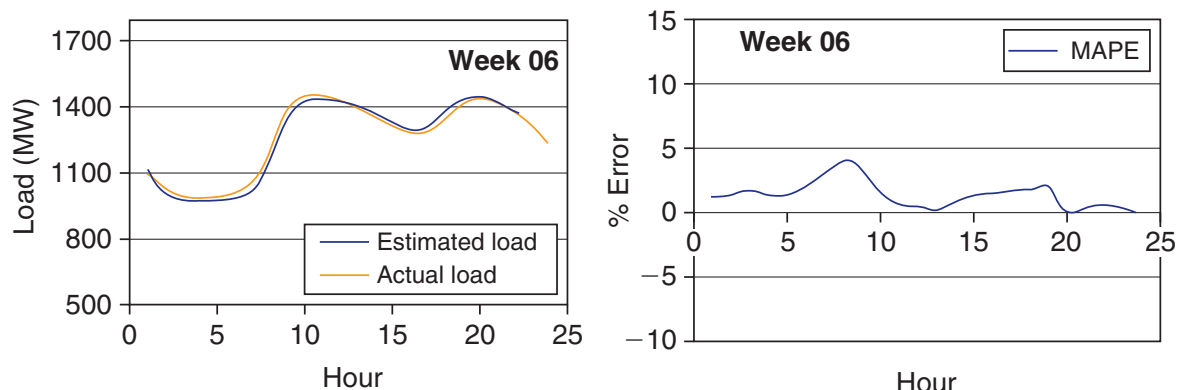

Figure 9.16 Estimated and actual load for 1995 during 24 hours.
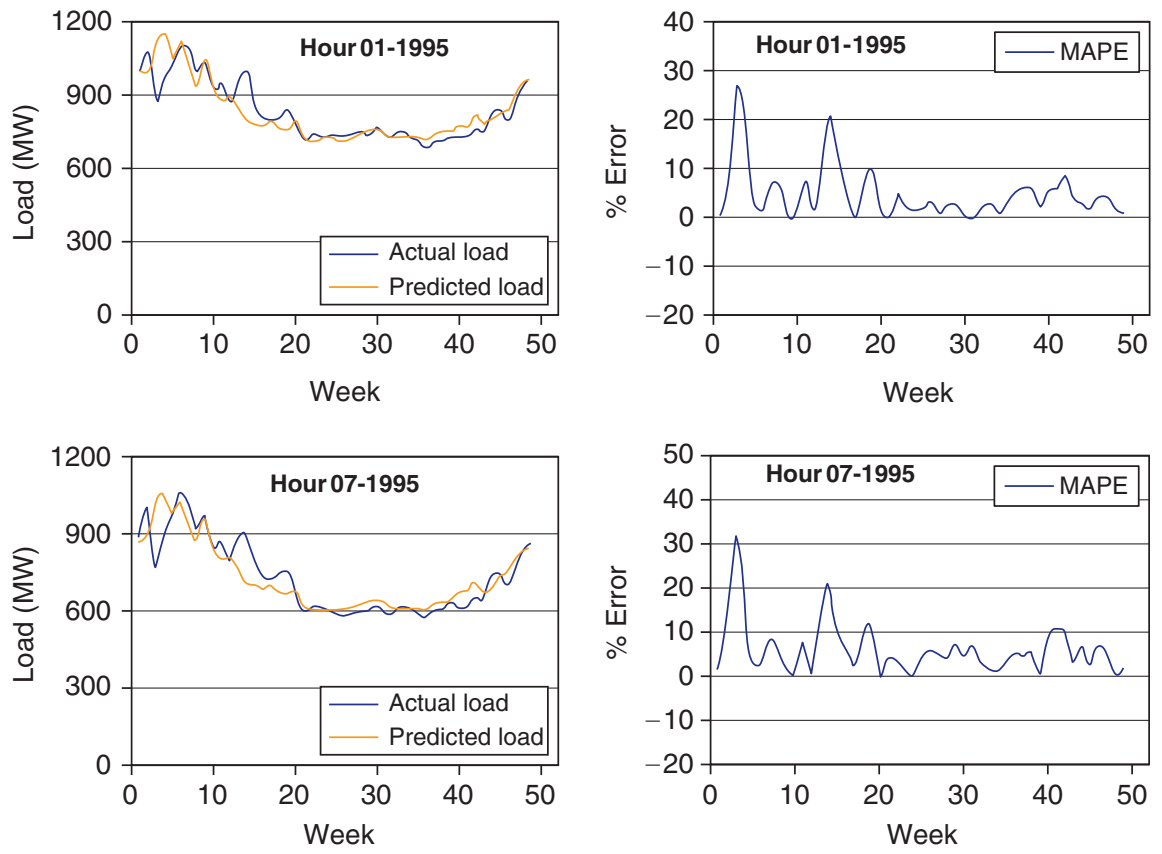

Figure 9.17 Estimated and actual loads for 1995 throughout 52 weeks. 
recursively to project the load behavior of the next years. The Kalman filtering algorithm exploits the annual load growth to effectively improve the forecasting accuracy. The results indicate that the mean absolute percentage error of the predicted daily load does not exceed $2.3 \%$ of the actual load over a whole year period. With the produced results, the proposed composite technique provides a significant advantage compared to those typically seen in the literature in increasing the forecast accuracy.

\subsection{Long-Term/Mid term Forecasting (Short-Term Correlation and Annual Growth)}

The great importance of long-term and mid term load forecasting for electric power utility planning and its economic consequences is encouraging the development of forecasting approaches in electric power research to improve its accuracy [20-36]. Since the 1980s, many techniques have been developed to improve long-term and mid term forecasting accuracy. Regression models utilize the strong correlation of load with load-affecting factors such as weather. A method of mathematical modeling for global forecasting based on regression analysis was used to forecast load demand up to 2000. Long-term forecasting based on linear and linear-log regression models of six predetermined sectors has been developed. The time-series models_-autoregressive (AR), moving average (MA), and autoregressive moving average (ARMA) — are popular and widely accepted by power utilities at present. They require a massive amount of historical data to produce optimal models. Gray system theory is successfully used to develop dynamic load-forecasting models.

By nature, long-term electric load forecasting is a complex problem. Among other factors, its accuracy is extremely influenced by the weather as well as social behavior of the community of that load. These factors are difficult to predict for the long-term load-forecasting time horizon. Conversely, short-term forecasting, though affected by weather and daily social habits, is small enough to predict load with high accuracy. Some short-term forecasting algorithms report to have results with a mean absolute error of less than $1 \%$. Consequently, short-term correlation of daily ( 24 hours) and yearly (52 weeks) load demand of a previous year is utilized to construct a one-year load-demand behavior. The load trends obtained thus far are adjusted with the annual load growth (ALG) to project load demand for the next year. Daily and yearly correlations are modeled as simple linear regressions on weekly average load (WAL) for the 24 hours and 52 weeks resulting in $(24 \times 52)$ simple linear regression equations. Daily regression is used to depict the relation between the loads at each hour with the hour prior to it, and weekly regression relates the average weekly load with the week prior to it.

\subsubsection{Load Regression Models}

The mid term and long-term electric load demand as a function of time has a complex nonlinear behavior. It depends on a number of complex factors such as daily and seasonal weather, national economic growth, and social habits. All these factors depend on time in a complex way. Therefore, a single mid term and long-term electric loaddemand model that accommodates most of these factors will have high nonlinear 
characteristics and may not give accurate prediction results. The approach taken in this section is the decomposition of the problem into multiple simple (first-order) linear regression models to capture the global nonlinear behavior of the load. Each of the linear regression models extracts the short-term correlation of a certain set of data. Then a recursive iterative algorithm is used to tie up the short-term results to capture the global load prediction.

One year's worth of data is arranged into a two-dimensional layout with 24 columns representing 24 hours of a day and 52 rows representing 52 weeks of the year. Figure 9.18 illustrates the 2D layout of the load data.

Special consideration is taken for the load variation during the weekends. Accordingly, weekends are treated separately but in the same manner exactly as the working days. The $L(i, k)$ cell in Figure 9.18 is the average load of the working days of the $i$ th week at the $k$ th hour. With this setup of the load data, obvious great intrinsic correlations exist between successive columns as well as between successive rows, as illustrated in Figures 9.19 and 9.20, respectively. These two figures and all subsequent results are based on the load demand of one of the largest electric power utilities in Canada for the years 1994 and 1995.

Figure 9.19 shows the load correlation between hour $1 \mathrm{AM}$ and hour $2 \mathrm{AM}$ throughout the whole year 1994. The correlation factor was calculated as 0.997. Similarly, Figure 9.20 shows the load correlation of week 1 and week 2 of year 1994, with a correlation factor of 0.985 . The strong correlation is maintained over the entire year for all 24 hours of the day, as illustrated in Figures 9.21 and 9.22.

The persistent correlation of the prevalent load patterns suggests the use of shortterm simple linear regression models for successive hours (see equation (9.24a)) and another set for successive weeks (see equation (9.24b)). This results in $24 \times 52$ simple linear regression models, which are used to draw the shape of the 2D load behavior contour for one year.

$$
\begin{aligned}
& L(i, k)=a(k) L(i, k-1)+b(k) \quad k=1, \ldots, 24 \\
& L(i, k)=c(i) L(i-1, k)+d(i) \quad i=1, \ldots, 52
\end{aligned}
$$

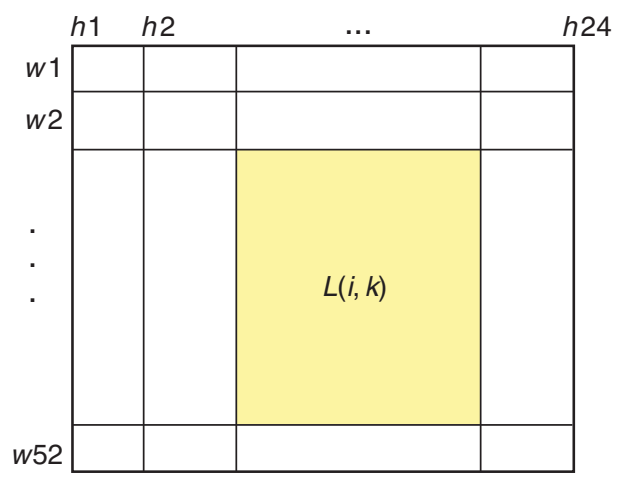

Figure 9.18 Two-dimensional layout of the load data. 


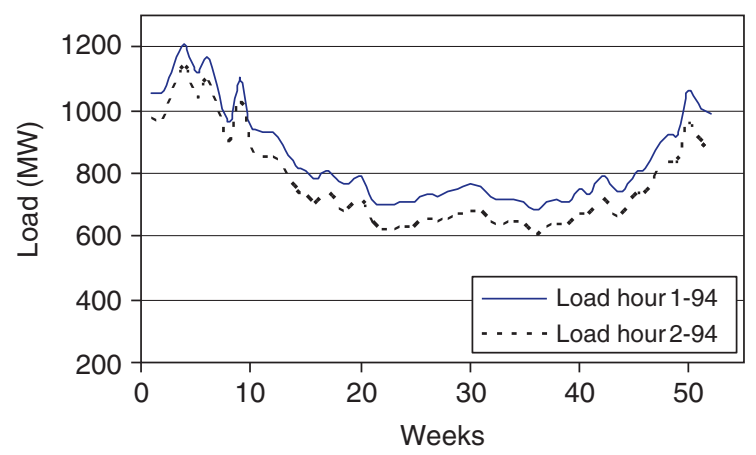

Figure 9.19 Comparing weekly average load of hours 1 and 2, 1994.

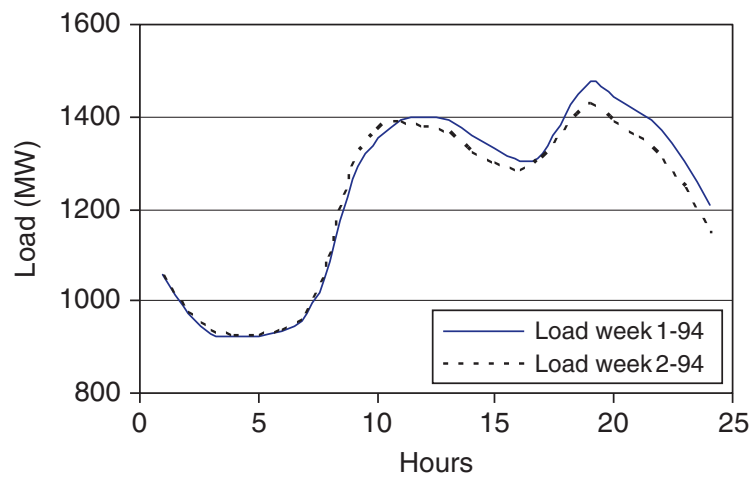

Figure 9.20 Comparing weekly average load of weeks 1 and 2, 1994.

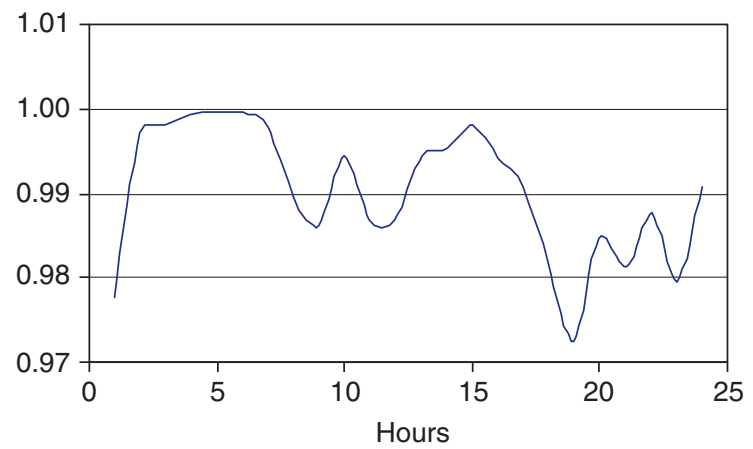

Figure 9.21 Correlation factor for successive hours over 52 weeks of 1994. 


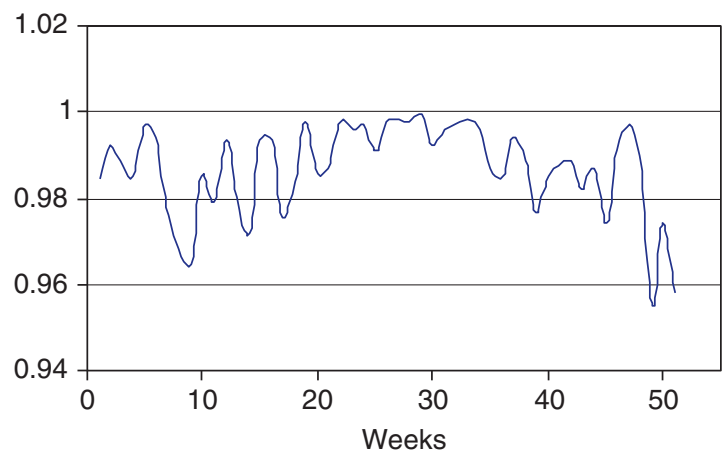

Figure 9.22 Correlation factor of successive weeks over 24 hours of 1994.

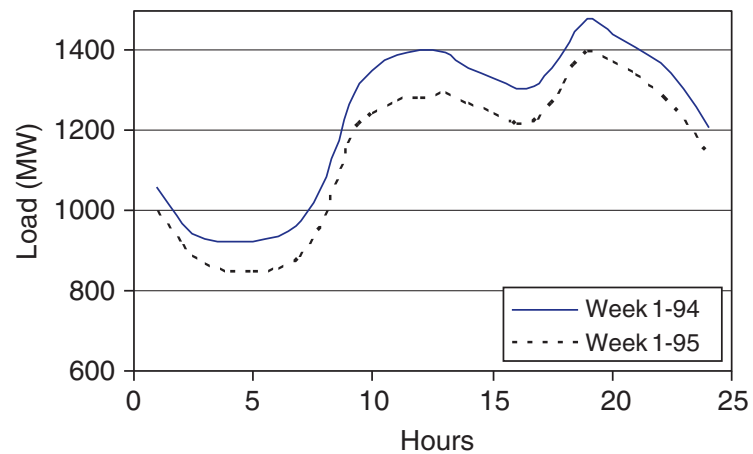

Figure 9.23 Comparing weekly average load of the first weeks of 1994 and 1995.

where

$a(k)$ and $b(k)$ are regression parameters at the $k$ th hour; $k=1,2, \ldots, 24$, which are estimated using the load pairs $[L(i, k), L(i, k-1)]$ for all $i=1,2, \ldots, 52$, by the least squares method; $L(i, k)$ and $L(i, k-1)$ are the weekly average load at hours $k$ and $k-1$, respectively, for all weeks $i=1, \ldots, 52$, with the initial condition $L(i, 0)=L(i-1,24)$;

$c(i)$ and $d(i)$ are regression parameters of the $i$ th week, $i=1,2, \ldots, 52$, which are estimated using the load pairs $[L(i, k), L(i-1, \mathrm{k})]$ for all $k=1,2, \ldots, 24$, by the least squares method; $L(i, k)$ and $L(i-1, k)$ are the weekly average load in $i$ th and $(i-1)$ th weeks, respectively, for all hours $k=1, \ldots, 24$, with the initial condition $L(0, k)=[L(52, k)$ of the previous year $]$.

\subsubsection{Estimating the Next Year's Load Contour}

The first-order regression models developed in the preceding section are used to project the load trends for the next year. Figures 9.23 and 9.24 demonstrate the fact that successive years have nearly identical load behavior contours. The load contours of the previous year (1994) coupled with the annual load growth are utilized to predict the next year's load (1995). Each regression model depicts a local relation of the load contours of the two years. The 24 linear regression models of equation (9.24a) relate 


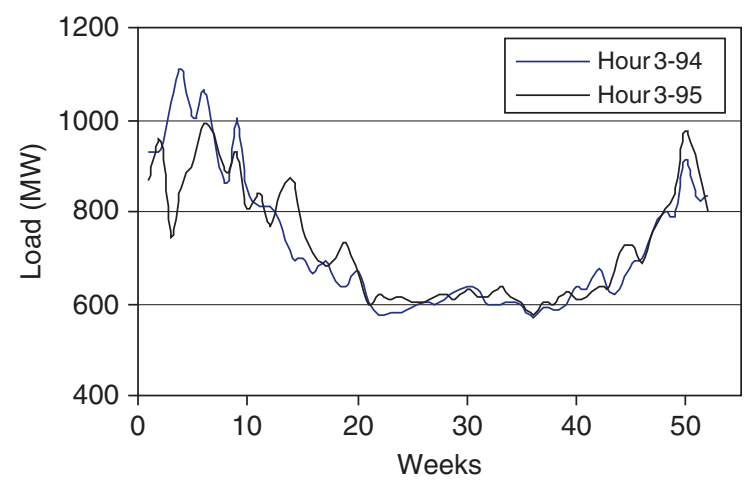

Figure 9.24 Comparing weekly average load of hour 3 of 1994 and 1995.

the load demand of successive hours of a day. They model the daily behavior of the load. The seasonal behavior of the load is modeled by the 52 linear regression models of equation (9.24b).

A recursive procedure used to estimate the next year's load contour utilizing regression models of the previous year is as follows:

1. Estimating for the first week the weekly average load: This process corresponds to estimating the first row of the next year's load; refer to Figure 9.25(a). Using equation (9.24b), we calculate $\hat{L}(1, k)$ :

$$
\hat{L}(1, k)=c(k) \hat{L}(0, k)+d(k) \quad k=1,2, \ldots, 24
$$

where $\hat{L}(1, k)$ is the estimated weekly average load of the first week at the $k$ th hour; $\hat{L}(1, k)$ is set to $L(52, k)_{\text {last-year }}$, which is the weekly average load of last year's 52 nd week; and $[c(k), d(k)]$ is a pair of regression coefficients of the $k$ th hour, obtained from equation (9.24b) using last year's data.

2. Estimating for the first hour the weekly average load: This process corresponds to estimating the first column of the next year's load; refer to Figure 9.25(b). Using equation (9.24a), we calculate $\hat{L}(i, 1)$ :

$$
\hat{L}(i, 1)=a(i) \hat{L}(i, 0)+b(i) \quad i=2,3, \ldots, 52
$$

where $\hat{L}(i, 1)$ is the estimated weekly average load of the first hour in the $i$ th week; $\hat{L}(i, 0)$ is set to $L(i-1,24)$, which is the weekly average load of the 24th hour of the previous week; and $[a(i), b(i)]$ is a pair of regression coefficients of the $i$ th week, obtained from equation (9.24a) using last year's data.

3. Estimating for the second week the weekly average load: This process corresponds to estimating the second row of the next year's load; refer to Figure 9.25(c). Using equation (9.24b), we calculate $\hat{L}(2, k)$ :

$$
\hat{L}(2, k)=c(k) \hat{L}(1, k)+d(k) \quad k=2,3, \ldots, 24
$$

where $\hat{L}(2, k)$ is the estimated weekly average load of the second week at the $k$ th hour, and $\hat{L}(2, k)$ is obtained using equation (9.25). 


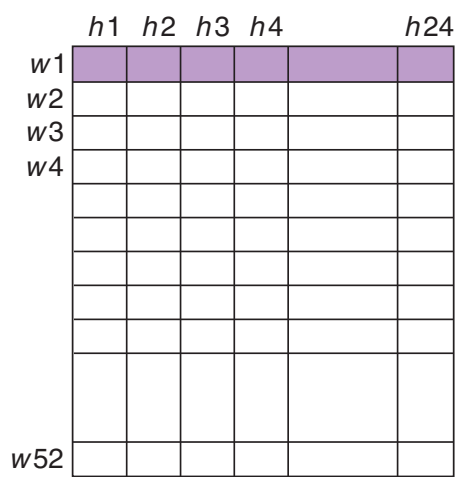

(a)

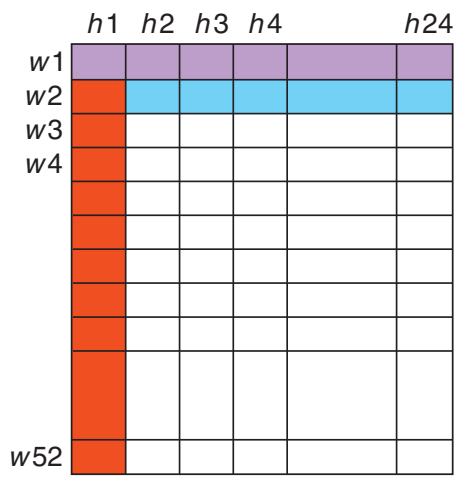

(c)

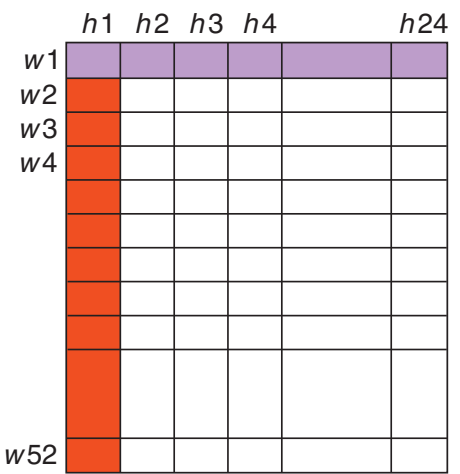

(b)

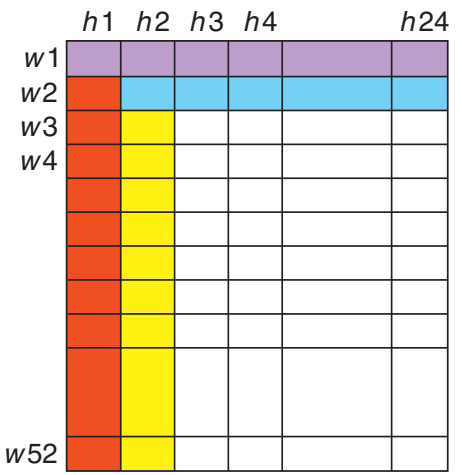

(d)

Figure 9.25 (a) First week (row) load estimation resulting from the first iteration. (b) First hour (column) load estimation resulting from the second iteration. (c) Second week (row) load estimation resulting from the third iteration. (d) Second hour (column) load estimation resulting from the fourth iteration.

4. Estimating for the second hour the weekly average load: This process corresponds to estimating the second column of the next year's load; refer to Figure 9.25(d). Using equation (9.24a), we calculate $\hat{L}(i, 2)$ :

$$
\hat{L}(i, 2)=a(i) \hat{L}(i, 1)+b(i) \quad i=3,4, \ldots, 52
$$

where $\hat{L}(i, 2)$ is the estimated weekly average load of the second hour in the $i$ th week and is obtained using equation (9.26).

5. The recursive iterations are repeated until $i=k=24$.

The preceding procedure produces a two-dimensional contour of the load behavior for one year based on regression coefficients of the previous year. The load contour will then be augmented by the annual load growth to account for the load change between successive years. 


\subsubsection{Annual Load Growth}

To maximize the accuracy of next year's load-demand estimation, we estimate and employ annual load growth as an adjusting factor. It is evident that load demand has a very strong dependence on time. Typical load profiles of successive years reveal very strong correlation at certain periodic time intervals. For example, refer to Figure 9.24; the two load curves at a certain hour over the whole year for two successive years retain the same shape. Moreover, there is, on average, a clear load increase over the previous year. This increase amounts to an annual load growth at that hour as a function of time (weeks) throughout the whole year. The load growth is modeled as the difference between the load curves of two successive years as a function of time.

Practical load profiles show that second-order models will not be sufficient to pick up the annual load growth variations. Third-order models or higher must be used. Models with orders 3, 4, 5, and 6 were tested to best fit load profiles. It was found that models with orders higher than third order were very sensitive to round-off errors and produce "very" incorrect results. A third-order polynomial is utilized to model the load as a function of time at the $k$ th hour as a function of the load of the previous hour. The regression model is as follows:

$$
L(i, k)=\beta_{0}(k)+\beta_{1}(k) L(i, k-1)+\beta_{2}(k) L^{2}(i, k-1)+\beta_{3}(k) L^{3}(i, k-1)
$$

where $\beta_{j}(k), j=0,1,2,3$, are regression variables at the $k$ th hour, and $k=1,2, \ldots, 24$, which are determined using the load pairs $[L(i, k), L(i, k-1)$, for all $i=1,2, \ldots, 52]$ by the least squares method. The initial values $L(i, 0)$ are set to $L(i-1,24)$. The two curves that approximate the relationship between $L(i, k)$ and $L(i, k-1)$ corresponding to the load behavior of the two years in Figure 9.24 are shown in Figure 9.26. The annual load growth curve is obtained by subtracting the approximate curve of 1995 (estimated data using regression models) from the approximate curve of 1994 (actual data), as shown in Figure 9.27.

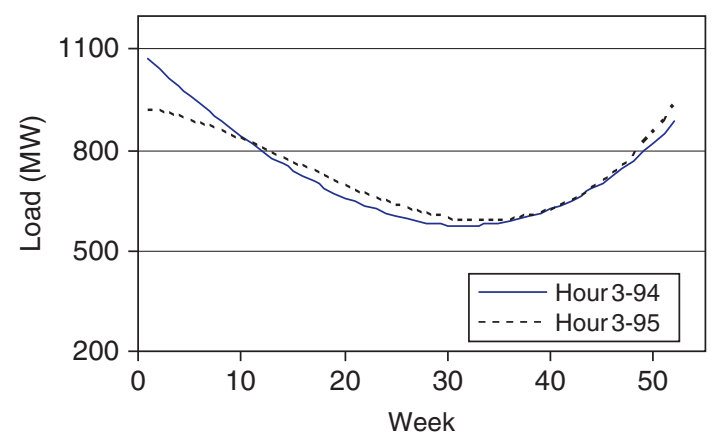

Figure 9.26 Approximate curves of load of third hour of 1994 and 1995. 


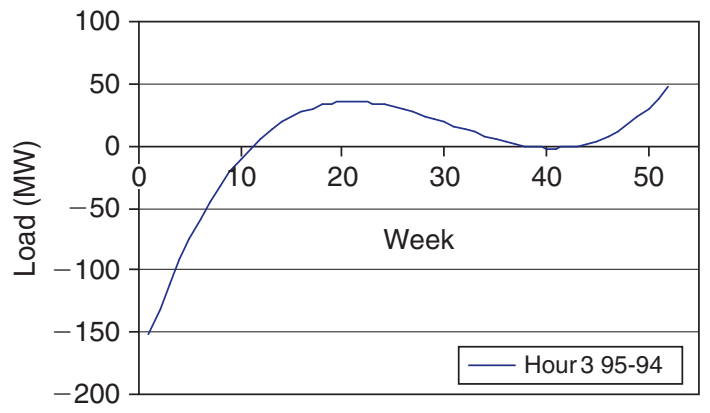

Figure 9.27 Annual load growth variations during 52 weeks of the year.

Next, the procedure for evaluating the annual load growth is as follows; we assume that the annual load growth is calculated between 1994 and 1995:

1. Using equation (9.29), we determine the regression coefficients ( 24 sets) for 24 hours for the actual date from year 1994. The coefficients define 24 approximate curves of the weekly average load, one curve per hour.

2. We repeat the calculations of the preceding step to the 1995 estimated data obtained using the regression models.

3. We define the annual load growth as the difference of the approximate load curves of 1995 and 1994 of steps 2 and 1, respectively:

$$
\begin{aligned}
& \text { Annual Load Growth }(i)=L(i, k)_{(95)}-L(i, k)_{(94)} \quad k=1,2, \ldots, 24 \text {, } \\
& i=1,2, \ldots, 52
\end{aligned}
$$

For each hour, the annual load growth is added to the 1995 estimated data obtained using the regression models to produce the final prediction results.

\subsection{Examples of Long-Term/Mid Term Forecasting}

To verify the effectiveness of the proposed load-demand forecasting technique, we used load data for one of the largest utility companies in Canada for the years 1994 and 1995. Regression models are obtained from 1994 data and used to project load demand for 1995.

\subsubsection{Multiple Regression Model Results}

Using equation (9.24a), we calculate 24 sets of regression coefficients. Table 9.13 shows the first seven of these sets as a sample. This table also lists the correlation factors of successive hours (columns) of the 1994 load data. Similarly, using equation (9.24b), we calculate 52 sets of regression coefficients. Table 9.14 shows the first seven of these sets as a sample, together with the correlation factors of successive weeks (rows) of the 1994 load data. 
Table 9.13 Correlation Factors and Regression Coefficients for Seven Hours of 1994

\begin{tabular}{|c|c|c|c|c|c|c|c|}
\hline 1994 & Hour 1 & Hour 2 & Hour 3 & Hour 4 & Hour 5 & Hour 6 & Hour 7 \\
\hline $\begin{array}{l}k=\text { hour of } \\
\text { the day }\end{array}$ & 1 & 2 & 3 & 4 & 5 & 6 & 7 \\
\hline \multicolumn{8}{|l|}{ Correlation } \\
\hline Factor & 0.978 & 0.997 & 0.998 & 0.999 & 0.999 & 1.000 & 0.998 \\
\hline$a(k)$ & 0.973 & 0.994 & 1.014 & 1.022 & 1.025 & 1.024 & 1.049 \\
\hline$b(k)$ & -89.311 & -76.835 & -49.053 & -31.009 & -21.580 & -11.659 & -6.003 \\
\hline
\end{tabular}

Table 9.14 Correlation Factors and Regression Coefficients of Seven Weeks of 1994

\begin{tabular}{llllr}
\hline 1994 & $\begin{array}{l}\boldsymbol{i} \text { =Week } \\
\text { Number }\end{array}$ & $\begin{array}{l}\text { Correlation } \\
\text { Factor }\end{array}$ & $\boldsymbol{c}_{(\boldsymbol{i})}$ & \multicolumn{1}{c}{$\boldsymbol{d}_{(\boldsymbol{i})}$} \\
\hline Week 1 & 1 & 0.985 & 0.918 & 80.911 \\
Week 2 & 2 & 0.993 & 0.964 & 137.674 \\
Week 3 & 3 & 0.987 & 0.953 & -86.209 \\
Week 4 & 4 & 0.985 & 0.983 & 43.987 \\
Week 5 & 5 & 0.997 & 1.025 & -5.718 \\
Week 6 & 6 & 0.994 & 0.909 & -252.143 \\
Week 7 & 7 & 0.976 & 1.161 & \\
\hline
\end{tabular}

\subsubsection{Estimating the 1995 Load Contour}

The MAPE with respect to the actual load is used to measure the effectiveness of the estimated results. For $n$ estimated load values, the MAPE error is given by the equation

$$
\text { MAPE }=\frac{100}{n} \sum_{i=1}^{n} \frac{\left|\hat{L}_{e s t, i}-L_{a c t, i}\right|}{L_{a c t, i}}
$$

where $\hat{L}_{e s t, i}$ and $L_{a c t, i}$ are the estimated and actual $i$ th load values, respectively. The recursive procedure outlined in Section 9.7.2 is used to project the shape of the 1995 load contour. The regression coefficients-namely $[c(i), d(i)]$ and $[a(k), b(k)]$ - are alternatively used to estimate a row and a column, respectively, of the 1995 contour described in Figure 9.18. The procedure is carried out for 24 iterations converging to the actual 1995 load. Figure 9.28(a) shows a sample of the MAPE error convergence for each hour over the 24 iterations. As shown, the error for each hour converges to its minimum. Figure 9.28(b) shows the convergence of the overall MAPE error for the whole year, which was found to be $5.12 \%$.

\subsubsection{Annual Load Growth Results}

The annual load growth is evaluated and used to augment the estimated load contours determined in Section 9.8.2. The third-order polynomial load models described in 


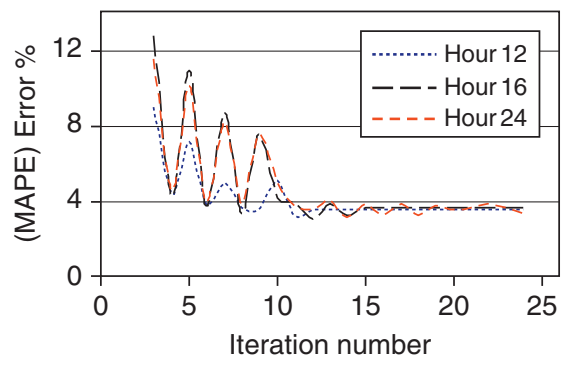

(a)

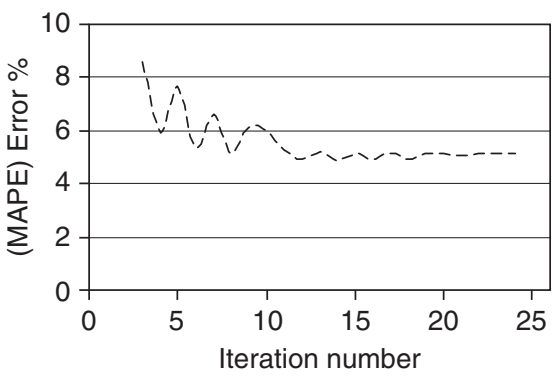

(b)

Figure 9.28 (a) Regression estimation (MAPE) error over 52 weeks of 1995. (b) Overall regression estimation (MAPE) error over 52 weeks of 1995.

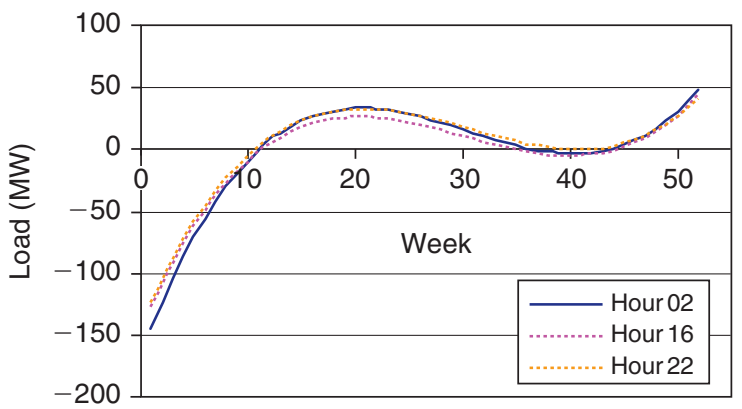

Figure 9.29 Annual load growth throughout 52 weeks of the year.

equation (9.28) are used to calculate the annual load growth for each hour of the day. Figure 9.26 shows the approximate fitted curves for hour 3 of 1994 and 1995, and Figure 9.27 shows the annual load growth for that hour. The annual load growth curves for all hours follow almost the same shape with very minimal variations, as illustrated by Figures 9.29 and 9.30. During almost the first 10 weeks, the annual load growth is negative. This accounts for the unexpectedly low load demand during these weeks in 1995, as shown in Figure 9.27. The low power consumption in these weeks of 1995 was mainly due to the above-normal high temperatures. The model naturally responds to the given data. It will react differently to different data from different utilities. To reduce the dependency of the annual load growth on uncontrollable short-term weather variations, we can calculate the average of the annual growth over several years.

Figure 9.31 shows a sample of the estimated weekly average load curves for some weeks together with MAPE error over 24 hours of the day. Similarly, Figure 9.32 presents a sample of the weekly average load for some hours varying over 52 weeks of the year. Introducing annual load growth improved the estimation results obtained in Section 9.8.2. The resulting overall MAPE is 3.8 with a standard deviation of 4.14. 


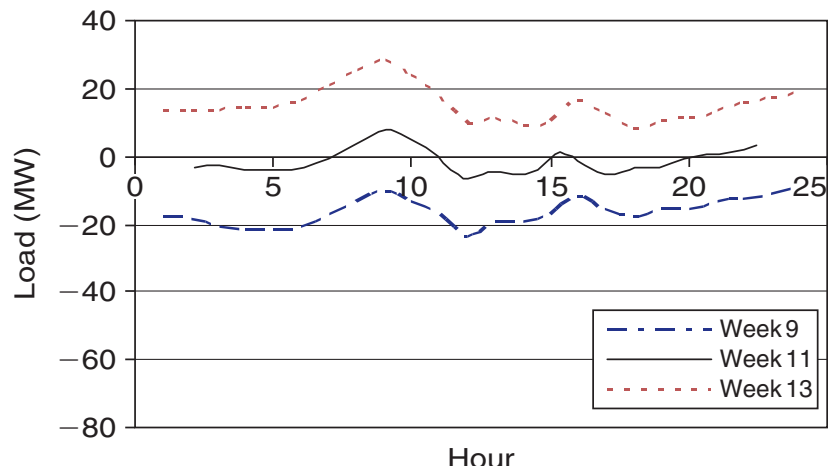

Figure 9.30 Annual growth variation during hours of a day.
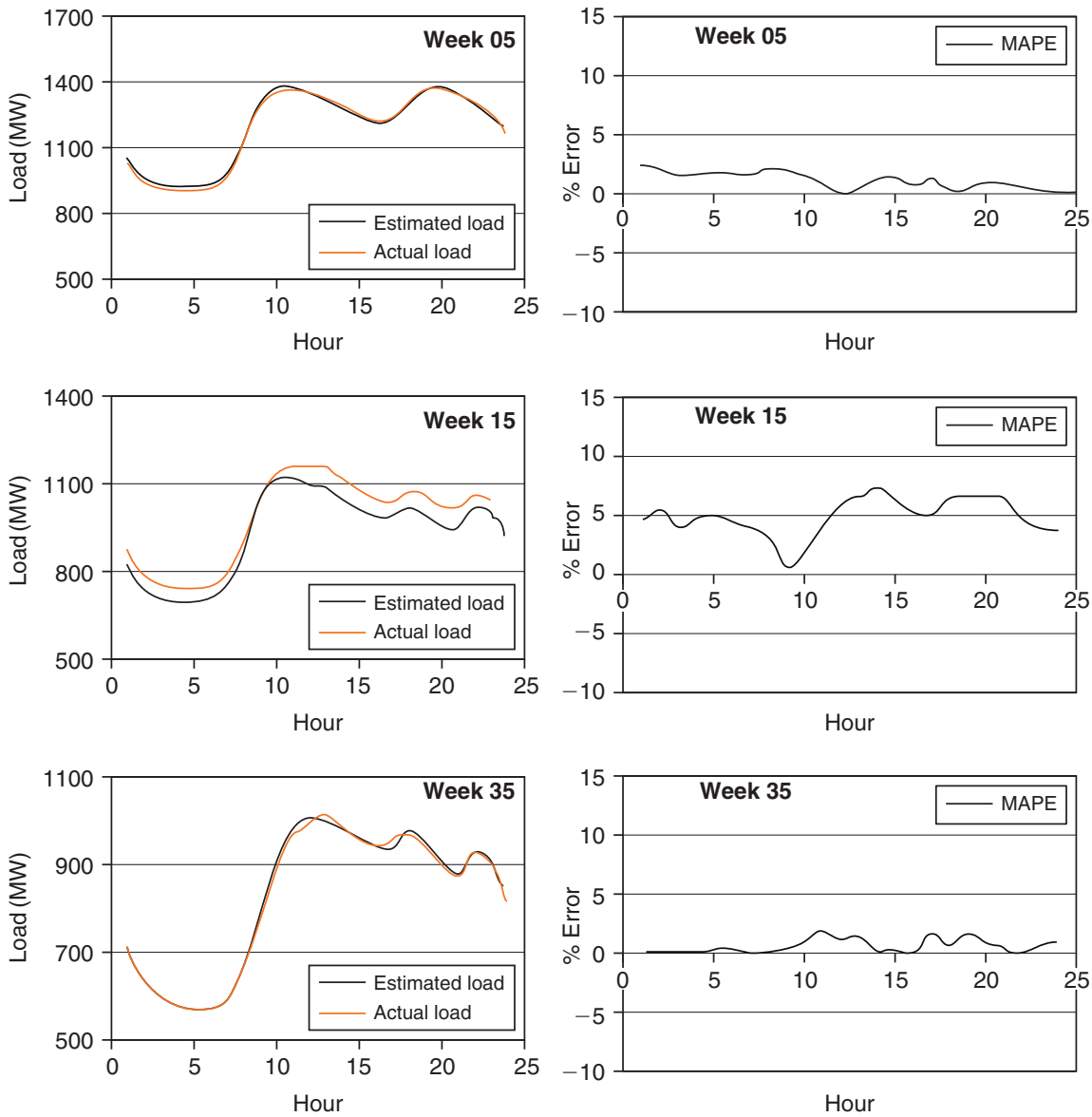

Figure 9.31 Comparison of a sample of estimated and actual load for 1995 during 24 hours. 

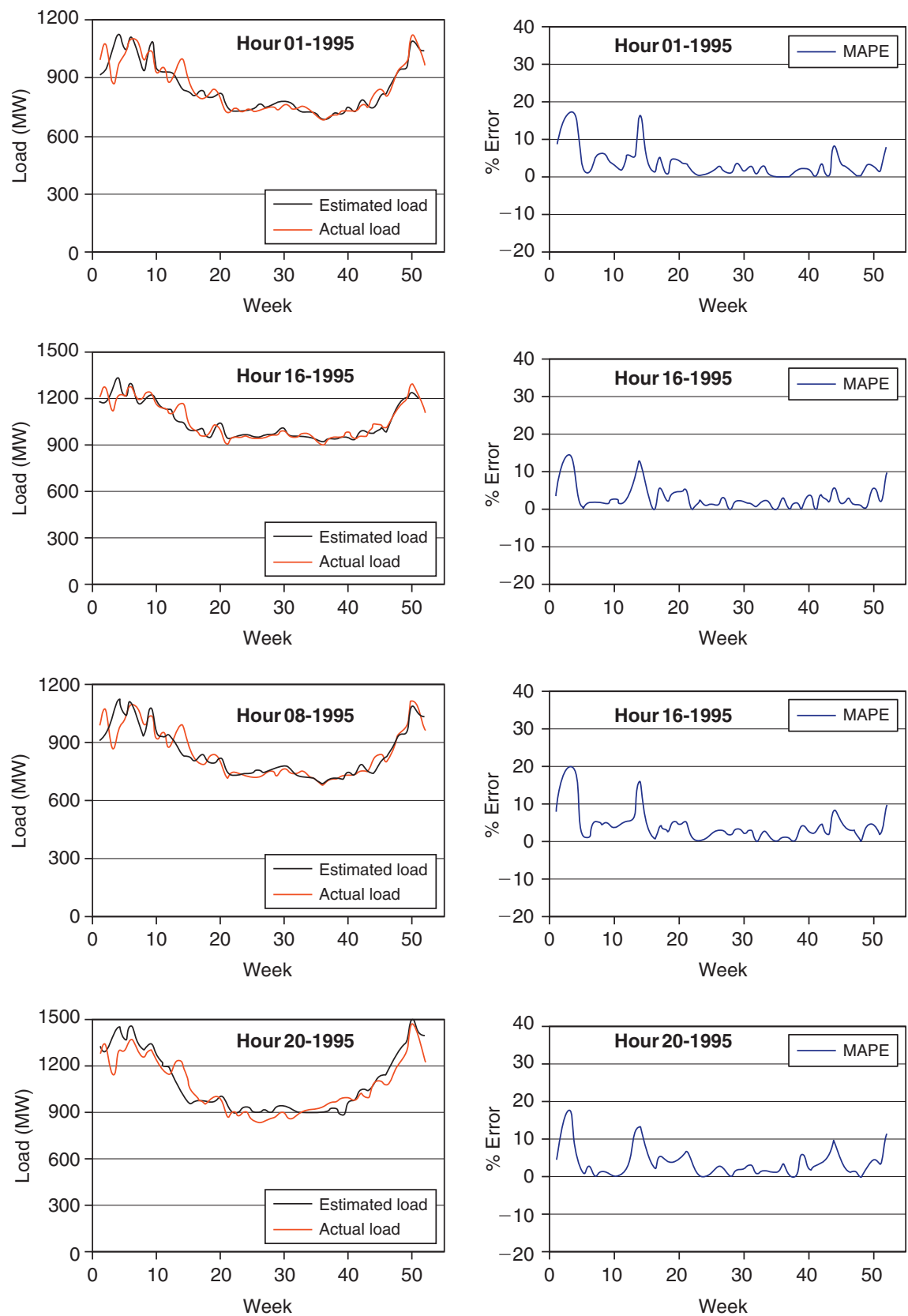

Figure 9.32 Comparison of a sample of estimated and actual loads for 1995 throughout 52 weeks. 


\subsubsection{Remarks}

This section demonstrated a long-term and mid term electric load-forecasting technique for forecasting hourly daily load demand for a lead time of several weeks to a few years. It was achieved utilizing short-term correlation of load behavior together with its annual growth. First, using historic data over a specific period of time (one year), we obtained the hourly daily load shape using multiple simple linear regression parametric load models. Second, we employed the parametric models obtained using alternating hourly and weekly load estimations to determine the shape of the load behavior for the next year. Last, we added annual growth load to correct the shape of the next year's load. The results indicated that the mean absolute error of the predicted weekly average daily load did not exceed $3.8 \%$ of the actual load over a whole year period. With the produced results, the proposed model and forecast technique used provide a significant advantage compared to those typically seen in the literature for reducing the average absolute error between the forecasted and actual loads over a forecast period of one year ahead.

\subsection{Fuzzy Regression for Peak-Load Forecasting}

In power system planning, a utility establishes goals and objectives, seeks to predict environmental factors, and then selects actions that result in the accomplishment of these goals and objectives [37-52]. The need for electric load forecasting is increasing as power system planning attempts to decrease its dependence on chance and becomes realistic in dealing with its environment.

Frequently, there is a time lag between awareness of an impending event or need and the occurrence of that event. This time lag is the main reason for power system planning and electric load forecasting. If the time lag is long and the outcome of the final event is conditional upon identifiable factors, power system planning can play an important role. In such situations, electric load forecasting is needed to determine when a need will arise so that the appropriate action can be taken.

The load growth of a geographical area served by a utility company is the most important factor influencing the expansion of a power system. Therefore, the forecasting of an increasing load and power system reaction to such load growth is essential to the planning process. Electric load forecasting can be regarded as answering this question: What amount of electricity should be arranged to supply a specific number and type of customer over a specific period of time? Forecasting can be achieved by performing analysis of past and/or present data, identifying trends and patterns that exist in the data that are then used to project load into the future.

This section presents the application of a fuzzy regression technique to long-term annual peak-load forecasting. The proposed technique takes into account the uncertainties in the nature of the peak load. Different factors are taken into account on modeling the peak load. These factors include the gross domestic product (GDP), population (POP), GDP/POP, the multiplication of the consumption of electricity and population (EP), the system losses (PL), and the rate of sale of electricity (RS; the price). Finally, we consider the time in question. Different fuzzy models are 
developed that relate these variables with the peak load. This section offers an example for estimating the peak load for the Egyptian Unified Network (EUN) to explain the main features of the proposed algorithm.

\subsubsection{Modeling of Electric Annual Peak Load}

Annual peak-load demand mainly depends on the community and the nation within this community. The main factors that greatly affect the growth of the load on a power system are different from one nation to another. For the Egyptian Unified Network, the following factors are to be considered when modeling the annual peak load:

- The gross domestic product (GDP)

- The population (POP)

- The gross domestic product per population (GDP/POP)

- The electric population (EP)

- The system losses (SL)

- The load factor (LF)

- The rate of sale (RS) measured in mill/kWh

- The time horizon (the year in question; T)

The annual peak-load demand is a function of these variables. The technique developed in reference [9] uses some of these factors to estimate the annual peakload demand of Japan. In this section, we consider all these variables to obtain a fuzzy model for the annual peak load.

\subsubsection{A Nonfuzzy Peak Load with Fuzzy Parameters}

In this section, we assume that the peak load is nonfuzzy, whereas the parameters of the load are fuzzy parameters with a symmetrical triangular membership function. In this case, the annual load model can be written as

$$
\begin{aligned}
P_{L}= & A_{0}+A_{1}(\mathrm{GDP})+A_{2}(\mathrm{POP})+A_{3}(\mathrm{EP})+A_{4}(\mathrm{GDP} / \mathrm{POP}) \\
& +A_{5}(\mathrm{SL})+A_{6}(\mathrm{LF})+A_{7}(\mathrm{RS})+A_{8}(\mathrm{~T})
\end{aligned}
$$

where $\underline{A}_{0}, \underline{A}_{2}, \ldots, \underline{A}_{8}$ are the model fuzzy parameters to be estimated, and each parameter has a certain middle $p$ and a certain spread $c$. Equation (9.32) can be rewritten as

$$
\begin{aligned}
P_{L}= & \left(p_{0}, c_{0}\right)+\left(p_{1}, c_{1}\right)(\mathrm{GDP})+\left(p_{2}, c_{2}\right)(\mathrm{POP})+\left(p_{3}, c_{3}\right)(\mathrm{EP}) \\
& +\left(p_{4}, c_{4}\right)(\mathrm{GDP} / \mathrm{POP})+\left(p_{5}, c_{5}\right)(\mathrm{SL})+\left(p_{6}, c_{6}\right)(\mathrm{LF}) \\
& +\left(p_{7}, c_{7}\right)(\mathrm{RS})+\left(p_{8}, c_{8}\right)(\mathrm{T})
\end{aligned}
$$

In fuzzy regression, we seek to find the fuzzy coefficients that minimize the spread of fuzzy output for all the data sets. In mathematical form, the objective function to be minimized is

$$
\begin{aligned}
O= & \min \left\{\sum _ { j = 1 } ^ { m } \left[c_{0}+c_{1}(\mathrm{GDP})_{j}+c_{2}(\mathrm{POP})_{j}+c_{3}(\mathrm{EP})_{j}+c_{4}(\mathrm{GDP} / \mathrm{CAP})_{j}\right.\right. \\
& \left.\left.+c_{5}(\mathrm{SL})_{j}+c_{6}(\mathrm{LF})_{j}+c_{7}(\mathrm{RS})_{j}+c_{8}(\mathrm{~T})_{j}\right]\right\}
\end{aligned}
$$


This is subject to satisfying two constraints on each annual peak-load demand as

$$
\begin{array}{rl}
\left(P_{L}\right)_{j} \geq & \left\{\left(p_{0}+p_{1}(\mathrm{GDP})_{j}+p_{2}(\mathrm{POP})_{j}+p_{3}(\mathrm{EP})_{j}+p_{4}(\mathrm{GDP} / \mathrm{CAP})_{j}+p_{5}(\mathrm{SL})_{j}\right.\right. \\
& \left.+p_{6}(\mathrm{LP})_{j}+p_{7}(\mathrm{RS})_{j}+p_{8}(\mathrm{~T})_{j}\right\}-(1-\lambda)\left\{\left(c_{0}+c_{1}(\mathrm{GDP})_{j}+c_{2}(\mathrm{POP})_{j}\right.\right. \\
& \left.+c_{3}(\mathrm{EP})_{j}+c_{4}(\mathrm{GDP} / \mathrm{CAP})_{j}+c_{5}(\mathrm{SL})_{j}+c_{6}(\mathrm{LP})_{j}+c_{7}(\mathrm{RS})_{j}+c_{8}(\mathrm{~T})_{j}\right\} \\
j=1, \ldots, m \\
\left(P_{L}\right)_{j} \leq & \left\{\left(p_{0}+p_{1}(\mathrm{GDP})_{j}+p_{2}(\mathrm{POP})_{j}+p_{3}(\mathrm{EP})_{j}+p_{4}(\mathrm{GDP} / \mathrm{CAP})_{j}+p_{5}(\mathrm{SL})_{j}\right.\right. \\
& \left.+p_{6}(\mathrm{LP})_{j}+p_{7}(\mathrm{RS})_{j}+p_{8}(\mathrm{~T})_{j}\right\}+(1-\lambda)\left\{\left(c_{0}+c_{1}(\mathrm{GDP})_{j}+c_{2}(\mathrm{POP})_{j}\right.\right. \\
& \left.\left.+c_{3}(\mathrm{EP})_{j}+c_{4}(\mathrm{GDP} / \mathrm{CAP})_{j}+c_{5}(\mathrm{SL})_{j}+c_{6}(\mathrm{LP})_{j}+c_{7}(\mathrm{RS})_{j}+c_{8}(\mathrm{~T})_{j}\right)\right\} \\
j=1, \ldots, m & j
\end{array}
$$

where $\lambda$ is the degree of fuzziness.

The problem formulated in equations (9.34) to (9.36) is a standard linear programming problem and can be solved using linear programming based on the simplex method available in the IMSL/STAT library.

Having identified the fuzzy parameters of the model, we could easily forecast the annual peak-load demand for any year, providing that the factors mentioned in Section 9.9.1 are available.

\subsubsection{A Fuzzy Peak-Load Demand}

Due to the uncertainties in the annual peak-load-demand forecasting, we assume that this load is a fuzzy load having a certain power $m_{j}$ with a spread $\alpha_{j}, j=1, \ldots, m$. In this case equation (9.32) can be rewritten as

$$
\begin{aligned}
\left(m_{j}, \alpha_{j}\right)= & \left(p_{0}, c_{0}\right)+\left(p_{1}, c_{1}\right)(\mathrm{GDP})_{j}+\left(p_{2}, c_{2}\right)(\mathrm{POP})_{j}+\left(p_{3}, c_{3}\right)(\mathrm{EP})_{j} \\
& +\left(p_{4}, c_{4}\right)(\mathrm{GDP} / \mathrm{POP})+\left(p_{5}, c_{5}\right)(\mathrm{SL})_{j}+\left(p_{6}, c_{6}\right)(\mathrm{LF})_{j} \\
& +\left(p_{7}, c_{7}\right)(\mathrm{RS})_{j}+\left(p_{8}, c_{8}\right)(\mathrm{T})_{j}
\end{aligned}
$$

Two fuzzy numbers are equal if and only if their middles and spreads are equal- that is

$$
\begin{aligned}
m_{j}= & p_{0}+p_{1}(\mathrm{GDP})_{j}+p_{2}(\mathrm{POP})_{j}+p_{3}(\mathrm{EP})_{j}+p_{4}(\mathrm{GDP} / \mathrm{POP})_{j} \\
& +p_{5}(\mathrm{SL})_{j}+p_{6}(\mathrm{LF})_{j}+p_{7}(\mathrm{RS})_{j}+p_{8}(\mathrm{~T})_{j} ; \quad j=1, \ldots, m
\end{aligned}
$$

and

$$
\begin{aligned}
\alpha_{j}= & c_{0}+c_{1}(\mathrm{GDP})_{j}+c_{2}(\mathrm{POP})_{j}+c_{3}(\mathrm{EP})_{j}+c_{4}(\mathrm{GDP} / \mathrm{POP})_{j} \\
& +c_{5}(\mathrm{SL})_{j}+c_{6}(\mathrm{LF})_{j}+c_{7}(\mathrm{RS})_{j}+c_{8}(\mathrm{~T})_{j} ; \quad j=1, \ldots, m
\end{aligned}
$$

The problem now turns out to be: Given the previous history of the fuzzy annual peak load in the form of $\left(m_{j}, \alpha_{j}\right)$, we need to find the fuzzy parameters $\underline{A}_{0}, \ldots, \underline{A}_{8}$ that minimize the cost function given by 


$$
\begin{aligned}
O= & \min \left\{\sum _ { j = 1 } ^ { m } \left[c_{0}+c_{1}(\mathrm{GDP})_{j}+c_{2}(\mathrm{POP})_{j}+c_{3}(\mathrm{EP})_{j}+c_{4}(\mathrm{GDP} / \mathrm{CAP})_{j}\right.\right. \\
& \left.+c_{5}(\mathrm{SL})_{j}+c_{6}(\mathrm{LF})_{j}+c_{7}(\mathrm{RS})_{j}+c_{8}(\mathrm{~T})_{j}\right\}
\end{aligned}
$$

This is subject to satisfying the following two constraints in each load power

$$
\begin{aligned}
m_{j}-(1-h) \alpha_{j} \geq & \left\{p_{0}+p_{1}(\mathrm{GDP})_{j}+p_{2}(\mathrm{POP})_{j}+p_{3}(\mathrm{EP})_{j}+p_{4}(\mathrm{GDP} / \mathrm{POP})_{j}\right. \\
& \left.+p_{5}(\mathrm{SL})_{j}+p_{6}(\mathrm{LF})_{j}+p_{7}(\mathrm{RS})_{j}+p_{8}(\mathrm{~T})_{j}\right\}-(1-\lambda)\left\{c_{0}\right. \\
& +c_{1}(\mathrm{GDP})_{j}+c_{2}(\mathrm{POP})_{j}+c_{3}(\mathrm{EP})_{j}+c_{4}(\mathrm{GDP} / \mathrm{POP})_{j} \\
& \left.+c_{5}(\mathrm{SL})_{j}+c_{6}(\mathrm{LF})_{j}+c_{7}(\mathrm{RS})_{j}+c_{8}(\mathrm{~T})_{j}\right\} ; \quad j=1, \ldots, m
\end{aligned}
$$

and

$$
\begin{aligned}
m_{j}+(1-h) \alpha_{j} \leq & \left\{p_{0}+p_{1}(\mathrm{GDP})_{j}+p_{2}(\mathrm{POP})_{j}+p_{3}(\mathrm{EP})_{j}+p_{4}(\mathrm{GDP} / \mathrm{POP}) j\right. \\
& \left.+p_{5}(\mathrm{SL})_{j}+p_{6}(\mathrm{LF})_{j}+p_{7}(\mathrm{RS})_{j}+p_{8}(\mathrm{~T})_{j}\right\}+(1-\lambda)\left\{c_{0}\right. \\
& +c_{1}(\mathrm{GDP})_{j}+c_{2}(\mathrm{POP})_{j}+c_{3}(\mathrm{EP})_{j}+c_{4}(\mathrm{GDP} / \mathrm{POP})_{j} \\
& \left.+c_{5}(\mathrm{SL})_{j}+c_{6}(\mathrm{LF})_{j}+c_{7}(\mathrm{RS})_{j}+c_{8}(\mathrm{~T})_{j}\right\}, \quad j=1, \ldots, m
\end{aligned}
$$

Again, the problem formulated in this section is a linear programming problem that can be solved using the simplex method.

Having identified the model fuzzy parameters, we can estimate the peak annual load for the forthcoming years.

\subsection{Testing the Algorithm}

\subsubsection{Nonfuzzy Annual Peak Load}

In this section we test the proposed algorithm for the data of the EUN [37-52]. The data are given in Table 9.15. The data from year 1981 to year $1992, T=0$ to $T=11$, are used to estimate the fuzzy parameter of the model given in equation (9.37). The unseen data for the rest of the years are used to evaluate the accuracy of the estimated parameters. The linear programming available in the IMSL/STAT library is used to solve the linear optimization problem. The fuzzy coefficients obtained are given as

$$
\begin{array}{ll}
\underline{A}_{0}=(0.0,222.382) & \underline{A}_{5}=(0.2652,0.0) \\
\underline{A}_{1}=(0.075,0.0) & \underline{A}_{6}=(0.0,0.0) \\
\underline{A}_{2}=(0.0,0.0) & \underline{A}_{7}=(0.0,0.0) \\
\underline{A}_{3}=(0.0,0.0) & \underline{A}_{8}=(154.135,0.0) \\
\underline{A}_{4}=(1.561,0.0) &
\end{array}
$$

Note that $\underline{A}_{0}$ is the only fuzzy parameter. These estimated parameters are used to estimate the annual peak load for the unseen data. Table 9.16 gives the results obtained. 
Table 9.15 Actual, Estimated Annual Peak Load

\begin{tabular}{lllll}
\hline Year & Actual Load & Estimated Load & Error (MW) & \% Error \\
\hline 1993 & 7503 & 7603.61 & -100.61 & -1.34 \\
1994 & 7657 & 7866.61 & -209.61 & -2.78 \\
1995 & 8149 & 8213.92 & -64.92 & -0.80 \\
1996 & 8491 & 8591.76 & -100.76 & -1.19 \\
\hline
\end{tabular}

Table 9.16 Estimated Parameters at Different Degrees of Fuzziness

\begin{tabular}{|c|c|c|c|}
\hline Parameter & $\lambda=0.25$ & $\lambda=0.5$ & $\lambda=0.75$ \\
\hline$\underline{A}_{0}$ & $(0,745)$ & $(0,805)$ & $(0,1013)$ \\
\hline$\underline{A}_{1}$ & $(0.165,0)$ & $(0.1337,0)$ & $(0.088,0)$ \\
\hline$\underline{A}_{2}$ & $(0,0)$ & $(7.985,0)$ & $(21.84,0)$ \\
\hline$\underline{A}_{3}$ & $(0,0)$ & $(0,0)$ & $(0,0)$ \\
\hline$\underline{A}_{4}$ & $(0,0)$ & $(0,0)$ & $(0,0)$ \\
\hline$\underline{A}_{5}^{+}$ & $(0.067,0)$ & $(0.11482,0)$ & $(0.179,0)$ \\
\hline$\underline{A}_{6}$ & $(0,0)$ & $(0,0)$ & $(0,0)$ \\
\hline$\underline{A}_{7}$ & $(0,0)$ & $(0,0)$ & $(0,0)$ \\
\hline$\underline{A}_{8}$ & $(108,0)$ & $(127.82,0)$ & $(153.5,0)$ \\
\hline
\end{tabular}

Examining Table 9.16 reveals that the proposed algorithm estimates the annual peak load very accurately, and the errors in the estimates are small compared to the other techniques described in the literature.

We examined the effects of the degree of fuzziness on the estimated parameters in this test, where we changed $\lambda$ from a small value, 0.0 , to a large value, 1.0. It has been found that the degree of fuzziness has no effect on the middle of the fuzzy coefficients, but as the degree of fuzziness increases, the spread of the output increases to satisfy the increased measure of goodness of fit.

\subsubsection{Fuzzy Annual Peak Load}

In this section we assume that the annual peak load is fuzzy and the spread of each measurement is 0.1 from the actual peak load given in Table 9.15. The problem formulated in equations (9.37), (9.38), and (9.39) is solved using the simplex method based on linear programming. Table 9.16 gives the estimated parameters at different degrees of fuzziness, for the first 12 measurements of Table 9.15. Tables 9.17 and 9.18 give the estimated load for the rest of the data of Table 9.15, unseen data, as well as the error in the estimated value.

Examining these tables reveals the following observations:

- All the parameters are nonfuzzy parameters except the first one.

- As the degree of fuzziness increases, the spread of $\underline{A}_{0}$ increases.

- The estimated load is very close to the actual load, even the spread of the load, and still the actual load moves between the boundaries of the triangular membership function we assumed. 
Table 9.17 Estimated Peak Load at $\lambda=0.25$

\begin{tabular}{lllr}
\hline Year & Actual $\boldsymbol{P}_{\boldsymbol{L}}$ & Estimated $\boldsymbol{P}_{\boldsymbol{l}}$ & \% Error \\
\hline 1993 & $(7503,750)$ & $(7498,745)$ & 0.060 \\
1994 & $(7657,766)$ & $(7824,745)$ & -2.200 \\
1995 & $(8149,815)$ & $(8217,745)$ & -0.083 \\
1996 & $(8491,849)$ & $(8647,745)$ & -9.943 \\
\hline
\end{tabular}

Table 9.18 Estimated Peak Load at $\lambda=0.5$

\begin{tabular}{llll}
\hline Year & Actual $\boldsymbol{P}_{\boldsymbol{L}}$ & Estimated $\boldsymbol{P}_{\boldsymbol{l}}$ & \% Error in the Estimates \\
\hline 1993 & $(7503,750)$ & $(7543,805)$ & -0.5 \\
1994 & $(7657,766)$ & $(7855,805)$ & -2.6 \\
1995 & $(8149,815)$ & $(8232,805)$ & -1.02 \\
1996 & $(8491,849)$ & $(8645,805)$ & -1.8 \\
\hline
\end{tabular}

- As $\lambda$ changes from 0.25 to 0.5 , the annual peak-load demand is changed, and having the same form as $\lambda$ changes from 0.5 to 0.75 . The degree of fuzziness has a great effect on the behavior of the model.

\subsubsection{Remarks}

In this section, we did the following:

- We developed a new fuzzy model for the annual peak-load demand for long-term planning.

- We developed models to solve the problem of uncertainties of the annual peak demand.

- We developed models to treat the long-term planning variables. Some of these variables depend on the nation of the community under investigation, whereas the others depend on the electric system itself.

- More investigation should be carried out to estimate the annual peak load for 15 or 20 years ahead. We were not able to do this due to the shortage of the data available to us.

\subsection{Time-Series Models}

A major aim of an electric power utility is to accurately forecast load requirements. In broad terms, power system load forecasting can be categorized into long-term and short-term functions [53-60]. Long-term load forecasting usually covers from 1 to 10 years ahead (monthly and yearly values) and is explicitly intended for applications in capacity expansion and long-term capital investment return studies.

In this section we mainly focus on the long-term load forecasting with mathematical methods. First, we introduce some basic foundations used with this forecasting. 


\subsubsection{Time Series}

A time series can be defined as a sequential set of data measured over time, such as hourly, daily, or weekly peak load. The basic idea of forecasting is to first build a pattern matching available data as accurately as possible and then obtain the forecasted value with respect to time using the established model.

Generally, series are often described as having the characteristic

$$
X(t)=T(t)+S(t)+R(t) \quad t=\ldots-1,0,1,2, \ldots
$$

where $T(t)$ is the trend term, $S(t)$ is the seasonal term, and $R(t)$ is the irregular or random component. At this point, we do not consider the cyclic terms because these fluctuations can have a duration from 2 to 10 years or even longer; therefore, they are not applicable to short-term load forecasting.

We assume the following to make this example a bit easier:

1. The trend is a constant level.

2. The seasonal effect has a period $s$; that is, it repeats after $s$ time periods. Or the sum of the seasonal components over a complete cycle or period is zero.

$$
\sum_{j=1}^{s} S(t+j)=0
$$

\subsubsection{Forecasting Methods}

To this point, we have used forecasting methods that are classified into two basic types: qualitative and quantitative methods.

Qualitative forecasting methods generally use the opinions of experts to predict future load subjectively. Such methods are useful when historical data are not available or are scarce. These methods include subjective curve fitting, the Delphi method, and technological comparisons.

Quantitative methods include regression analysis, decomposition methods, exponential smoothing, and the Box-Jenkins methodology.

\subsubsection{Forecasting Errors}

Unfortunately, all forecasting situations involve some degree of uncertainty, which makes errors unavoidable.

The forecast error for a particular forecast $\hat{X}_{t}$ with respect to actual value $X_{t}$ is

$$
e_{t}=X_{t}-\hat{X}_{t}
$$

To avoid the offset of positive with negative errors, we need to use absolute deviations:

$$
\left|e_{t}\right|=\left|X_{t}-\hat{X}_{t}\right|
$$


Hence, we can define a measure known as the mean absolute deviation (MAD) as follows:

$$
\mathrm{MAD}=\frac{\sum_{t=1}^{n}\left|e_{t}\right|}{n}=\frac{\sum_{t=1}^{n}\left|X_{t}-\hat{X}_{t}\right|}{n}
$$

Another method is to use the mean square error (MSE) defined as follows:

$$
\mathrm{MSE}=\frac{\sum_{t=1}^{n} e_{t}{ }^{2}}{n}=\frac{\sum_{t=1}^{n}\left(X_{t}-\hat{X}_{t}\right)^{2}}{n}
$$

\subsection{Power System Load Forecasting}

The power system load is assumed to be time dependent, evolving according to a probabilistic law [58]. It is common practice to employ a white noise sequence as input to a linear filter of which the output is the power system load. This is an adequate model for predicting the load time series. The noise input is assumed normally distributed, with zero mean and some variance $\sigma^{2}$. A number of classes of models exist for characterizing the linear filter.

\subsubsection{A Simple Example of Power System Load Forecasting}

Consider the data on fuel consumption given in Table 9.19.

We can average the seasonal values over the series and use these, minus the overall mean, as seasonal estimates shown here (overall mean is 761.65):

$$
\begin{array}{ll}
S(1)=888.2-761.65=126.55 & S(2)=709.2-761.65=-52.4 \\
S(3)=616.4-761.65=-145.25 & S(4)=832.8-761.65=71.15
\end{array}
$$

After subtraction of these values, the original series removes seasonal effects. It should be noted that this technique works well on series having linear trends with small slopes.

Table 9.19 Primary Energy Consumption in a Utility (Coal Equivalent)

\begin{tabular}{lrrrr}
\hline Year & \multicolumn{1}{l}{ 1 } & \multicolumn{2}{l}{$\mathbf{3}$} & \multicolumn{1}{c}{$\mathbf{4}$} \\
\hline 1965 & 874 & 679 & 616 & 816 \\
1966 & 866 & 700 & 603 & 814 \\
1967 & 843 & 719 & 594 & 819 \\
1968 & 906 & 703 & 634 & 844 \\
1969 & 952 & 745 & 635 & 871 \\
Means & 888.2 & 709.2 & 616.4 & 832.8 \\
\hline
\end{tabular}


In addition, we can look at the averages for each complete seasonal cycle (the period) because the seasonal effect over an entire period is zero. To avoid losing too much data, we use a method called moving average (MA), which is simply the series of averages:

$$
\frac{1}{s} \sum_{j=0}^{s-1} X_{t+j}, \frac{1}{s} \sum_{j=1}^{s} X_{t+j}, \frac{1}{s} \sum_{j=2}^{s+1} X_{t+j}, \ldots
$$

A problem is presented here if the period is even because the adjusted series values do not correspond to the original ones at time points. To overcome this problem, we use the centered moving average (CMA) to bring us back to the correct time points. This CMA is shown in Table 9.20.

Through looking at the differences between CMA and the original series, we can estimate the $k$ th seasonal effect simply by average the $k$ th quarter differences:

$$
S(1)=128.954 \quad S(2)=-46.487 \quad S(3)=-142 \quad S(4)=65
$$

But the sum of these four values is 5.107. Recall that we assume the seasonal sum to be zero, so we need to add a correction factor of $-5.107 / 4=-1.254$ to give

$$
S(1)=127.34 \quad S(2)=-47.741 \quad S(3)=-143.254 \quad S(4)=63.746
$$

\begin{tabular}{|c|c|c|c|c|}
\hline Quarter & $X(t)$ & MA & CMA(Order-2) & Difference \\
\hline 1 & 874 & & & \\
\hline 2 & 679 & & & \\
\hline 3 & 616 & 746.25 & 745.25 & -129.25 \\
\hline 4 & 816 & 744.25 & 746.875 & 69.125 \\
\hline 5 & 866 & 749.5 & 747.875 & 118.125 \\
\hline 6 & 700 & 746.25 & 746 & -46 \\
\hline 7 & 603 & 745.75 & 741.75 & -138.75 \\
\hline 8 & 814 & 737.75 & 740.125 & 73.875 \\
\hline 9 & 834 & 742.5 & 741.375 & 92.625 \\
\hline 10 & 719 & 740.25 & 740.875 & -21.875 \\
\hline 11 & 594 & 741.5 & 750.5 & -156.5 \\
\hline 12 & 819 & 759.5 & 757.5 & 61.5 \\
\hline 13 & 906 & 755.5 & 760.5 & 145.5 \\
\hline 14 & 703 & 765.5 & 768.625 & -65.625 \\
\hline 15 & 634 & 771.75 & 777.5 & -143.5 \\
\hline 16 & 844 & 783.25 & 788.5 & 55.5 \\
\hline 17 & 952 & 793.75 & 793.875 & 158.125 \\
\hline 18 & 745 & 794 & 797.375 & -52.375 \\
\hline 19 & 635 & 800.75 & & \\
\hline 20 & 871 & & & \\
\hline
\end{tabular}

Table 9.20 Calculation of the Moving Average 
Table 9.21 Prediction of Energy Consumption

\begin{tabular}{lccll}
\hline Period $\boldsymbol{t}$ & Trend $\boldsymbol{T}$ & Seasonal $\boldsymbol{S}$ & Predicted & Actual $\boldsymbol{X}$ \\
\hline 21 & 789.963 & 127.340 & 917.303 & 981 \\
22 & 793.610 & -47.741 & 747.123 & 759 \\
23 & 797.257 & -143.254 & 654.003 & 674 \\
24 & 800.904 & 63.746 & 864.650 & 900 \\
\hline
\end{tabular}

Now the irregular component can be easily calculated by subtracting both the CMA and the seasonal effects.

If we suppose the model (1) is appropriate, then we can use it to make predictions. To simplify, we omit the random data, so all we need to do is to predict the trend, say, a linear trend:

$$
T(t)=a+b t
$$

With the application to the CMA, we have

$$
\hat{T}(t)=713.376+3.647 t
$$

Hence, a prediction is shown in Table 9.21.

\subsection{Linear Regression Method}

The linear regression method is already used in short-term load forecasting and supposes that the load is affected by some factors such as high and low temperatures, weather condition, economic growth, etc. This relation can be expressed as

$$
y=\beta_{0}+\beta_{1} x_{1}+\beta_{2} x_{2}+\cdots+\beta_{k} x_{k}+\varepsilon
$$

where $y$ is the load, $x_{i}$ is the affecting factors, $\beta_{i}$ are regression parameters with respect to $x_{i}$, and $\varepsilon$ is an error term.

For this model, we always assume that the error term $\varepsilon$ has a mean value equal to zero and constant variance.

Since parameters $\beta_{i}$ are unknown, they should be estimated from observations of $y$ and $x_{i}$. Let $b_{i}(i=0,1,2, \ldots, k)$ be the estimates in terms of $\beta_{i}(i=0,1,2, \ldots, k)$. Recall that the error term has a 50\% chance of being positive and negative, respectively, so we omit this term in calculating parameters, which means

$$
\hat{y}=b_{0}+b_{1} x_{1}+b_{2} x_{2}+\cdots+b_{k} x_{k}
$$

Then, we use the least error squares estimate method, which minimizes the sum of squared residuals (SSE), to obtain the parameters $b_{i}$

$$
\underline{B}=\left[\begin{array}{llll}
b_{0} & b_{1} & b_{2} & \ldots \\
b_{k}
\end{array}\right]^{T}=\left(\underline{X}^{T} \underline{X}\right)^{-1} \underline{X}^{T} \underline{Y}
$$


where $\underline{Y}$ and $\underline{X}$ are the following column vector and matrix:

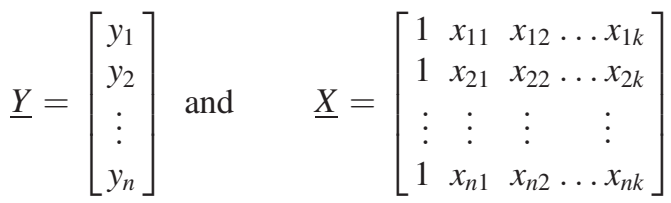

After the parameters are calculated, this model can be used for prediction. It will be accurate in predicting $y$ values if the standard error $s$ is small:

$$
s=\sqrt{\frac{S S E}{n-(k+1)}}, \quad S S E=\sum_{i=1}^{n}\left(y_{i}-\hat{y}_{i}\right)^{2}, \quad y_{i}: \text { observed, } \quad \hat{y}_{i}: \text { estimated }
$$

There are also some other ways to check the validity of a regression model [1].

\subsection{Autoregressive (AR) Model}

In the autoregressive model, the current value $X_{t}$ of the time series is expressed linearly in terms of its previous values $X_{t-1}, X_{t-2}, \ldots$ and a white noise series $\left\{\varepsilon_{t}\right\}$ with zero mean and variance $\sigma^{2}$ :

$$
X_{t}=\phi_{1} X_{t-1}+\phi_{2} X_{t-2}+\cdots+\phi_{p} X_{t-p}+\varepsilon_{t}
$$

By introducing the backshift operator $B$ that defines $X_{t-1}=B X_{t}$, and consequently $X_{t-m}=B^{m} X_{t}$, we can rewrite equation (9.54) in the form

$$
\phi(B) X_{t}=\varepsilon_{t}
$$

where

$$
\phi(B)=1-\phi_{1} B-\phi_{2} B^{2}-\cdots-\phi_{p} B^{p}
$$

Note that this model has a similar form to the multiple linear regression models. The difference is that in regression the variable of interest is regressed onto a linear function of other known (explanatory) variables, whereas here $X_{t}$ is expressed as a linear function of its own past values - thus, the description "autoregressive." As the values of $X_{t}$ at $p$ previous times are involved in the model, it is said to be an $\operatorname{AR}(p)$ model.

Now we need to calculate the parameters $\varphi_{i}$ for prediction. There are two such methods: least squares estimation and maximum likelihood estimation (MLE).

To calculate the least squares estimators, we need to minimize the expression (here, we let $p=2$ )

$$
\sum_{t=1}^{N}\left(X_{t}-\phi_{1} X_{t-1}-\phi_{2} X_{t-2}\right)^{2}
$$


with respect to $\varphi_{1}$ and $\varphi_{2}$. But because we do not have the information for $t=1$ or $t=2$, an assumption is made here that $X_{1}$ and $X_{2}$ are fixed, and excluding the first two terms from the sum of squares. That is, to minimize

$$
\sum_{t=3}^{N}\left(X_{t}-\phi_{1} X_{t-1}-\phi_{2} X_{t-2}\right)^{2}
$$

then we use a similar approach to linear regression to obtain the parameters.

Maximum likelihood estimation is attractive because generally it is asymptotically unbiased and has minimum variance. Therefore, we introduce this method here.

Suppose that we have a sample of dependent observations $X_{t}, t=1, \ldots, N$, each with $f\left(X_{t}\right)$. Then the joint density function is

$$
f\left(X_{1}, X_{2}, \ldots, X_{N}\right)=\prod_{t=1}^{N} f\left(X_{t} \mid \underline{X}_{t-1}\right)
$$

where $\underline{X}_{t}$ denotes all observations up to and including $X_{t} . f\left(X_{t} \mid \underline{X}_{t-1}\right)$ is the conditional distribution of $X_{t}$ given all observations prior to $t$.

We use the same model as before and suppose $\varepsilon_{t}$ is normally distributed. So the mean of the conditional distribution is $\phi_{1} X_{t-1}+\phi_{2} X_{t-2}$, and the variance is $\sigma^{2}$. Therefore,

$$
f\left(X_{t} \mid X_{1}, X_{2}, \ldots, X_{N}\right)=\frac{1}{\sqrt{(2 \pi)} \sigma} \exp \left[\frac{-\left(X_{t}-\phi_{1} X_{t-1}-\phi_{2} X_{t-2}\right)^{2}}{2 \sigma^{2}}\right]
$$

Similarly, we set $X_{1}$ and $X_{2}$ to be fixed and define the conditional likelihood as

$$
L(\theta)=\prod_{t=3}^{N} f\left(X_{t} \mid X_{1}, X_{2}, \ldots, X_{t-1}\right)
$$

By minimizing $L(\theta)$, we can obtain the parameters.

Consider a time series of the number of reported purse snatchings in a particular area 28 days apart, as shown in Figure 9.33.

If we use the MLE applied to the AR (2) model, the fitted model is

$$
X_{t}=0.0307841 X_{t-1}+0.400178 X_{t-2}+\varepsilon_{t} \operatorname{var}\left(\varepsilon_{t}\right)=36.115343
$$

Now, this model can be used to predict future data.

\subsection{Moving Average (MA) Model}

In the moving average process, the current value of the time series $X_{t}$ is expressed linearly in terms of current and previous values of a white noise series $\varepsilon_{t}, \varepsilon_{t-1}, \ldots$. This noise series is constructed from the forecast errors or residuals when load observations become available. The order of this process depends on the oldest noise value 


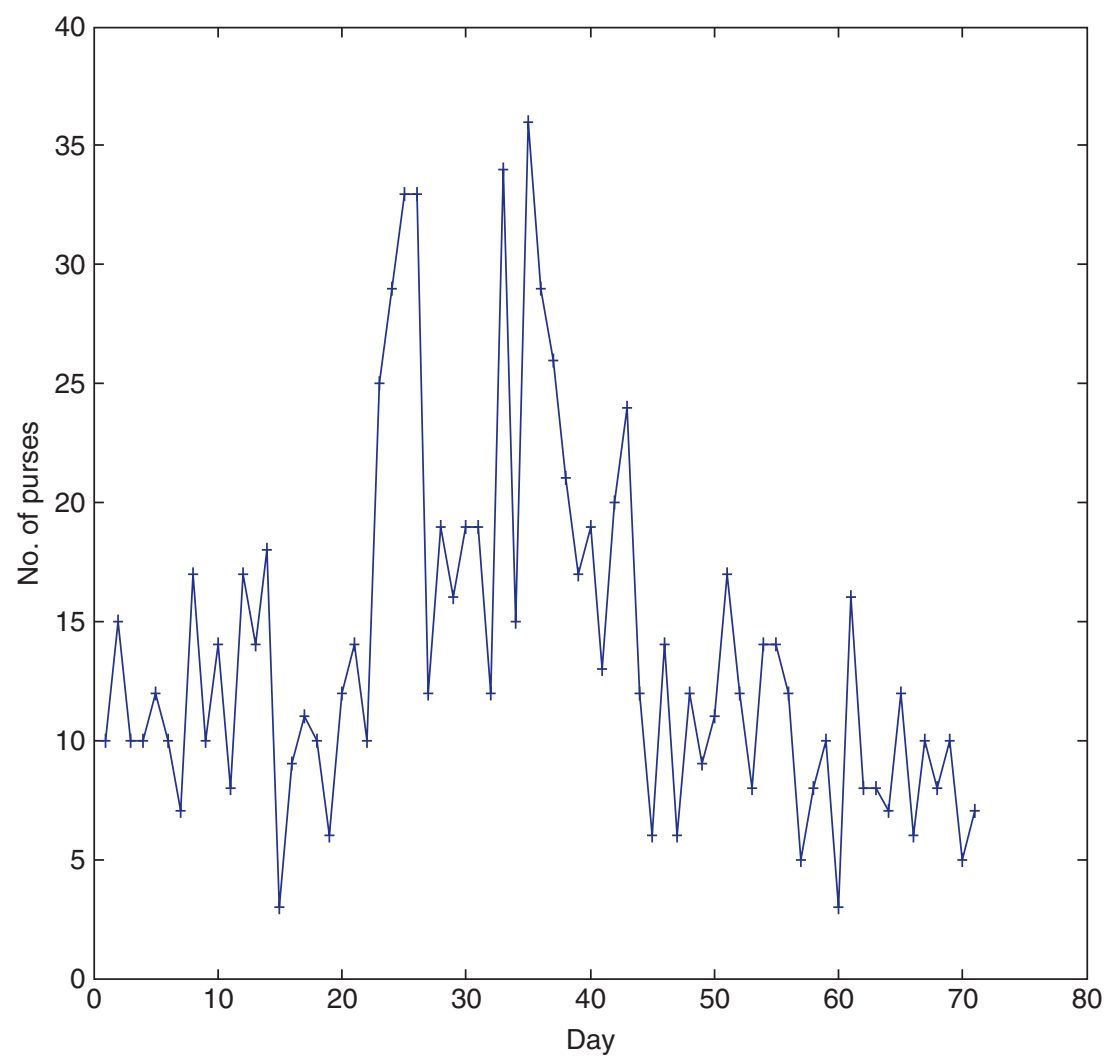

Figure 9.33 Reported purse snatching in an area.

at which $X_{t}$ is regressed. For a moving average of order $q$ (i.e., $M A(q)$ ), this model can be written as

$$
X_{t}=\varepsilon_{t}-\theta_{1} \varepsilon_{t-1}-\theta_{2} \varepsilon_{t-2}-\cdots-\theta_{q} \varepsilon_{t-q}
$$

A similar application of the backshift operator on the white noise series would allow equation (9.61) to be rewritten as

$$
X_{t}=\theta(B) \varepsilon_{t}
$$

where $\theta(B)=1-\theta_{1} B-\theta_{2} B^{2}-\cdots-\theta_{q} B^{q}$.

\subsection{Autoregressive Moving Average (ARMA, or Box-Jenkins) Model}

If we combine the MA and AR models, we can present a broader class of model-that is the autoregressive moving average model-as 


$$
X_{t}=\phi_{1} X_{t-1}+\phi_{2} X_{t-2}+\cdots+\phi_{p} X_{t-p}+\varepsilon_{t}+\theta_{1} \varepsilon_{t-1}+\theta_{2} \varepsilon_{t-2}+\cdots+\theta_{q} \varepsilon_{t-q}
$$

where $\phi_{i}$ and $\theta_{j}$ are called the autoregressive and moving average parameters, respectively. And in this case, this is an $\operatorname{ARMA}(p, q)$ model.

A methodology for ARMA models was developed largely by Box and Jenkins [60] so the models are often called Box-Jenkins models.

\subsection{Autoregressive Integrated Moving Average (ARIMA) Model}

The time series defined previously as an AR, MA, or ARMA process is called a stationary process [6]. This means that the mean of the series of any of these processes and the covariance among its observations do not change with time. Unfortunately, this is not often true in a power load. But previous knowledge is definitely useful in that the nonstationary series can be transformed into a stationary one with some tricks. This transformation can be achieved, for the time series that are nonstationary in the mean, by a differencing process. By introducing the $\nabla$ operator, we can write a differenced time series of order one as

$$
\nabla X_{t}=X_{t}-X_{t-1}=(1-B) X_{t}
$$

Consequently, an order $d$ differenced time series is written as

$$
\nabla^{d} X_{t}=(1-B)^{d} X_{t}
$$

The differenced stationary series can be modeled as AR, MA, or ARMA to yield an ARI, IMA, or ARIMA time-series process. For a series that needs to be differenced $d$ times and has orders $p$ and $q$ for the $\operatorname{AR}$ and MA components (i.e., $\operatorname{ARIMA}(p, d, q)$ ), the model is written as

$$
\phi(B) \nabla^{d} X_{t}=\theta(B) \varepsilon_{t}
$$

However, as a result of daily, weekly, yearly, or other periodicities, many time series exhibit periodic behaviors in response to one or more of these periodicities. Therefore, a seasonal ARIMA model is appropriate. It has been shown that the general multiplicative model $(p, d, q) *(P, D, Q)_{s}$ for a time-series model can be written in the form

$$
\phi(B) \Phi\left(B^{S}\right) \nabla^{d} \nabla_{S}^{D} X_{t}=\theta(B) \Theta\left(B^{S}\right) \varepsilon_{t}
$$

where definitions for $\Phi\left(B^{S}\right), \nabla_{S}^{D}, \Theta\left(B^{S}\right)$ are given in the following:

$$
\begin{aligned}
& \nabla_{S}^{D}=\left(X_{t}-X_{t-S}\right)^{D}=\left(1-B^{S}\right)^{D} X_{t} \\
& \Phi\left(B^{S}\right)=1-\Phi_{1} B^{S}-\Phi_{2} B^{2 S}-\cdots-\Phi_{p} B^{p S} \\
& \Theta\left(B^{S}\right)=1-\Theta_{1} B^{S}-\Theta_{2} B^{2 S}-\cdots-\Theta_{q} B^{q S}
\end{aligned}
$$


The model presented in equation (9.67) can obviously be extended to the case in which data for two seasons are accounted for. An example demonstrating seasonal time-series modeling is the model for hourly load data with a daily cycle. Such a model can be expressed using the model of equation (9.67) with $S=24$.

To obtain this model, we use the parameters $p, d, q, P, D, Q$, and other coefficients. By studying the self-variance, covariance, and variance function of the order one or higherorder differentiation of variables we get the $d$ and $D$. Then the model can be simplified into AR, MA, or ARMA models to calculate other values so that the model can be built.

\subsection{ARMAX and ARIMAX Models}

ARMA and ARIMA use only the time and load as input parameters. Because load generally depends on the weather and time of the day, exogenous variables sometimes can be included to give the ARMAX and ARIMAX models [7]. Other useful methods implementing evolutionary programming (EP) and fuzzy logic (FL) into conventional timeseries models were also proposed. We will not consider these methods in detail here.

\subsubsection{Remarks}

No one method can be applicable to all situations. So a method should be chosen considering many factors, such as the time frame, pattern of data, cost of forecasting, desired accuracy, availability of data, and ease of operation and understanding. Therefore, more work still needs to be done to include all these factors.

\section{References}

[1] H.L. Willis, L.A. Finley, M.J. Buri, Forecasting electric demand of distribution system in rural and sparsely populated regions, IEEE Trans. Power Syst. 10 (4) (1995) 2008-2013.

[2] P.H. Henault, R.B. Eastvedt, J. Peschon, L.P. Hajdu, Power system long term planning in the presence of uncertainty, IEEE Trans. Power Apparatus Syst. PAS-89 (1970) 156-164.

[3] G.S. Christensen, A. Rouhi, S.A. Soliman, A new technique for unconstrained and constrained LAV parameter estimation, Can. J. Elect. Comp. Eng. 14 (1) (1989) 24-30.

[4] Ministry of Electricity and Energy, Egyptian Electricity Authority, Load and energy forecast for the period 1996/1997 to 2009/2010, Report, February 1998.

[5] H.K. Temraz, K.M. El-Nagar, M.M.A. Salama, Applications of non-iterative least absolute value estimation for forecasting annual peak electric power demand, Can. J. Elect. Comp. Eng. 23 (4) (1998) 141-146.

[6] D. Srinivasan, T.S. Swee, C.S. Cheng, E.K. Chan, Parallel neural network-fuzzy expert system strategy for short-term load forecasting: system implementation and performance evaluation, IEEE Trans. Power Syst. 14 (3) (1999) 1100-1106.

[7] I. Drezga, S. Rahman, Short-term load forecasting with local ANN predictors, IEEE Trans. Power Syst. 14 (3) (1999) 844-850.

[8] A.A. Ding, Neural-network prediction with noisy predictors, IEEE Trans. Neural Netw. 10 (5) (1999) 1196-1203. 
[9] H.C. Wu, C. Lu, Automatic fuzzy model identification for short term load forecast, IEE Proc. Gener. Transm. Distrib. C 146 (5) (1999) 477-482.

[10] W. Charytoniuk, M.S. Chen, Very short-term load forecasting using artificial neural networks, IEEE Trans. Power Syst. 15 (1) (2000) 263-268.

[11] K.H. Kim, H.S. Youn, Y.C. Kang, Short-term forecasting for special days in anomalous load conditions using neural networks and fuzzy inference method, IEEE Trans. Power Syst. 15 (2) (2000) 559-565.

[12] P.A. da Silva, L.S. Moulin, Confidence intervals for neural network based short-term load forecasting, IEEE Trans. Power Syst. 15 (4) (2000) 1191-1196.

[13] S.A. Villalba, C.A. Bel, Hybrid demand model for load estimation and short-term load forecasting in distribution electric systems, IEEE Trans. Power Syst. 15 (2) (2000) 764-769.

[14] R.H. Liang, C.C. Cheng, Combined regression-fuzzy approach for short-term load forecasting, IEE Proc. Gener. Transm. Distrib. 147 (4) (2000) 261-266.

[15] H.S. Hippert, C.E. Pedreira, R.C. Souza, Neural networks for short-term load forecasting: a review and evaluation, IEEE Trans. Power Syst. 16 (1) (2001) 44-55.

[16] M. Huang, H.T. Yang, Evolving wavelet-based networks for short-term load forecasting, IEE Proc. Gener. Transm. Distrib. 148 (3) (2001) 222-228.

[17] D. Srinivasan, M.A. Lee, Survey of hybrid fuzzy neural approach to electric load forecasting, IEEE Int. Conf. Syst. Man Cybern. 5 (1995) 4004-4008.

[18] F.K. Selker, W.R. Wroblewski, Medium-term load forecasting and wholesale transaction profitability, IEEE Trans. Power Eng. Soc. (summer meeting) (1990) 1268-1272.

[19] N.X. Jia, R. Yokoyama, Y.C. Zuou, Z.Y. Gao, A flexible long-term forecasting approach based on a new dynamic simulation theory-GSIM, Int. J. Electr Power Energ. Syst. 23 (2001) 549-556.

[20] V.M. Vlahovic, I.M. Vujosevic, Long-term forecasting: a critical review of direct-trend extrapolation methods, Int. J. Electr. Power Energ. Syst. 9 (1) (1987) 2-8.

[21] E. Handchin, Ch. Dornemann, Bus load modeling and forecasting, IEEE Trans. Power Syst. 3 (2) (1988) 627-633.

[22] A.G. Parlos, E. Oufi, J. Muthusami, A.D. Patton, Development of an intelligent longterm electric load forecasting system, in: Proceedings, ISAP '96, International Conference, 28 Jan.-2 Feb 1996. Intell. Syst. Appl. Power Syst. (1996) 288-292.

[23] E.H. Barakat, S.A. Al-Rashid, Long-term peak demand forecasting under conditions of high growth, IEEE Trans. Power Syst. 7 (4) (1992) 1483-1486.

[24] M.R. Gent, Electric supply and demand in the US: next 10 years, IEEE Power Eng. Rev. 12 (4) (1992) 8-13.

[25] S.C. Terpathy, Demand forecasting in a power system, Energ. Conv. Manage. 38 (14) (1997) $1475-1481$.

[26] Y. Minato, Y. Yokoi, Development of a forecasting method of a region's electric power demand (1)-forecasting economic and social indices, Tans. IEE Jpn 116B (1996) 147-154.

[27] S. Tomonobu, S. Hirokazu, T. Yoshinori, U. Katsumi, Next-day load curve forecasting using neural network based on similarity, Electr. Power Compon. Syst. 29 (2001) 939-948.

[28] H.M. Al-Hamadi, S.A. Soliman, Short-term electric load forecasting based on Kalman filtering algorithm with moving windows weather and load model, Electr. Power Syst. Res. 68 (1) (2004) 47-59.

[29] R.G. Brown, Introduction to Random Signal Analysis and Kalman Filtering, John Wiley and Sons, New York, 1983.

[30] G.F. Franklin, J.D. Powel, M.L. Workman, Digital Control of Dynamic Systems, second ed. Addison-Wesley, Reading, MA, 1990. 
[31] C.W. Fu, T.T. Nguyen, Models for long-term energy forecasting, IEEE Power Eng. Soc. Gen. Meeting 1 (2003) 235-239.

[32] K. Nagasaka, M. Al Mamun, Long-term peak demand prediction of 9 Japanese power utilities using radial basis function networks, IEEE Power Eng. Soc. Gen. Meeting 1 (2004) 315-322.

[33] S. Phimphachanh, K. Chamnongthai, P. Kumhom, A. Sangswang, Using neural network for long term peak load forecasting in Vientiane municipality, TENCON 2004, 2004 Region 10 Conference, C, (2004) 147-154.

[34] B. Kermanshahi, H. Iwamiya, Up to 2020 load forecasting using neural nets, Int. J. Electr. Power Energ. Syst. 24 (2002) 789-797.

[35] X. Da, Y. Jiangyan, Y. Jilai, The physical series algorithm of mid-long term load forecasting of power systems, Electr. Power Res. J. 53 (2000) 31-37.

[36] M.S. Kandil, S.M. El-Debeiky, N.E. Hasanien, Overview and comparison of longterm forecasting techniques for fast developing utilities: Part I, Electr. Power Res. J. 58 (2001) 11-17.

[37] M.S. Kandil, S.M. El-Debeiky, N.E. Hasanien, The implementation of long term forecasting strategies using a knowledge-based expert system: Part II, Electr. Power Res. J. 58 (2001) 19-25.

[38] D. Srinivasan, M.A. Lee, Survey of hybrid fuzzy neural approach to electric load forecasting, IEEE Trans. Power Syst. 5 (1995) 4004-4008.

[39] V.M. Vlahovic, I.M. Vujosevic, Long-term forecasting: a critical review of direct-trend extrapolation methods, Electr. Power Energ. Syst. 9 (1) (1987) 2-8.

[40] L. Chenhui, Theory and Methods of Load Forecasting of Power Systems, Haerbin Institute of Technology Press, China, 1987.

[41] Y. Tamura, Z. Deping, N. Umeda, K. Sakashita, Load forecasting using grey dynamic model, IEEE Trans. Power Syst. (1995) 361-365.

[42] V.M. Vlahovic, I.M. Vujosevic, Long-term forecasting: a critical review of direct-trend extrapolation methods, Electr. Power Energ. Syst. 9 (1) (1987) 2-8.

[43] D.W. Bunn, E.D. Farmer (Eds.), Comparative Models for Electric Load Forecasting, John Wiley and Sons, Hoboken, NJ, 1985.

[44] H.K. Temraz, V.H. Quintana, Analytic spatial electric load forecasting methods: a survey, Can. J. Elect. Comp. Eng. 17 (1) (1992) 34-41.

[45] J. Nazarko, W. Zalewski, The fuzzy regression approach to peak load estimation in power distribution systems, IEEE Trans. Power Syst. 14 (3) (1999) 809-813.

[46] J.R. Yokoyama, Y.C. Zhou, A novel approach to long term load forecasting where functional relations and impact relations coexist. Int. Conf. Elect. Power Tech., Budapest, August 29-September 2, 1999.

[47] S.K. Padmakumari, K.P. Mohandas, D. Thiruvengadam, Application of fuzzy system theory in land use based long-term distribution load forecasting, in: Proceedings of EMPD'98, IEEE Catalog Number 98EX137, Int. Conf. on Energy Management and Power Delivery, 311-316.

[48] H.K. Temraz, K.M. El-Naggar, M.M. Salama, Application of noniterative least absolute value estimation for forecasting annual peak electric load power demand, Can. J. Elect. Comp. Eng. 23 (4) (1998) 141-146.

[49] A.G. Parlos, E. Oufi, J. Muthusami, A.D. Patton, A.F. Atiya, Development of an intelligent long term electric load forecasting system, in: Proceedings of ISAP'96, IEEE Catalog Number 96TH8152, Int. Conf. on Intelligent Systems Applications to Power Systems (1996) 288-292.

[50] A.G. Parlos, A.D. Patton, Long-term electric load forecasting using a dynamic neural network architecture, Joint Int. Power Conference, Athens Power Tech'1993, APT'93, 816-820. 
[51] P.Y. Wang, G.S. Wang, Power system load forecasting with ANN and fuzzy logic control, Int. Conf. on Computer Communication, Control and Power Engineering, TENCON'93, IEEE Catalog Number 93CH3286-2, 5, 359-362.

[52] E.H. Barakat, J.M. Al-Qassim, S.A. Al Rashed, New model for peak demand forecasting applied to highly complex load characteristics of fast developing area, IEE Proc. C 139 (2) (1992) 136-140.

[53] B.L. Bowerman, R.T. O'Connell, A.B. Koehler, Forecasting, Time Series, and Regression: An Applied Approach, fourth ed., Thomson Brooks/Cole, Duxbury, CA, 2005.

[54] D.C. Montgomery, L.A. Johnson, J.S. Gardiner, Forecasting \& time series analysis, second ed., McGraw-Hill, New York, 1990.

[55] G.A.N. Mbamalu, M.E. El-Hawary, Load forecasting via suboptimal seasonal autoregressive models and iteratively reweighted least squares estimation, IEEE Trans. Power Syst. 8 (1) (1993) 343-348.

[56] J.H. Chow, F.F. Wu, J.A. Momoh, Applied Mathematics for Restructured Electric Power Systems, Springer, New York, 2005.

[57] G. Janacek, L. Swift, Time Series: Forecasting, Simulation, Applications, Ellis Horwood Limited, West Sussex, 1993.

[58] I. Moghram, S. Rahman, Analysis and evaluation of five short-term load forecasting techniques, IEEE Trans. Power Syst. 4 (4) (1989) 1484-1491.

[59] J.Y. Fan, J.D. McDonald, A real-time implementation of short-term load forecasting for distribution power systems, IEEE Trans. Power Syst. 9 (2) (1994) 988-994.

[60] G.E.P. Box, G.M. Jenkins, G.C. Reinsel, Time Series Analysis, Forecasting and Control, third ed., Prentice Hall, Englewood Clifs, NJ, 1994. 